\title{
Euler type linear and half-linear differential equations and their non-oscillation in the critical oscillation case
}

\author{
Zuzana Došlál* ${ }^{*}$ Petr Hasil', Serena Matucci and Michal Veselý1
}

\section{"Correspondence:}

dosla@math.muni.cz

'Department of Mathematics and

Statistics, Faculty of Science,

Masaryk University, Brno, Czech

Republic

Full list of author information is

available at the end of the article

\begin{abstract}
This paper is devoted to the analysis of the oscillatory behavior of Euler type linear and half-linear differential equations. We focus on the so-called conditional oscillation, where there exists a borderline between oscillatory and non-oscillatory equations. The most complicated problem involved in the theory of conditionally oscillatory equations is to decide whether the equations from the given class are oscillatory or non-oscillatory in the threshold case. In this paper, we answer this question via a combination of the Riccati and Prüfer technique. Note that the obtained non-oscillation of the studied equations is important in solving boundary value problems on non-compact intervals and that the obtained results are new even in the linear case.
\end{abstract}

MSC: $34 \mathrm{C} 10 ; 34 \mathrm{C} 15$

Keywords: Half-linear equations; Linear equations; Euler type equations; Oscillation theory; Oscillation criterion; Non-oscillation criterion; Oscillation constant; $p$-Laplacian

\section{Introduction}

In this paper, we study oscillatory properties of the half-linear differential equation

$$
\left(r(t) \Phi\left(x^{\prime}\right)\right)^{\prime}+s(t) \Phi(x)=0, \quad \Phi(x)=|x|^{p-1} \operatorname{sgn} x, \quad p>1,
$$

where $r$ and $s$ are continuous functions and $r$ is positive. In fact, we focus on Eq. (1.1) in the so-called Euler form. Euler type differential equations play an important role in solving non-linear BVP associated to equations with $p$-Laplacian (see, e.g., $[6,8,9]$ and the references therein). Our research is also motivated by paper [3], in which an eigenvalue problem associated to the half-linear equation

$$
\left(r(t) \Phi\left(x^{\prime}\right)\right)^{\prime}+\left(s_{1}(t)+\lambda s_{2}(t)\right) \Phi(x)=0
$$

is studied, and by [24, 48], in which the oscillation of a neutral half-linear differential equation is examined.

(c) The Author(s) 2019. This article is distributed under the terms of the Creative Commons Attribution 4.0 International License (http://creativecommons.org/licenses/by/4.0/), which permits unrestricted use, distribution, and reproduction in any medium, provided you give appropriate credit to the original author(s) and the source, provide a link to the Creative Commons license, and indicate if changes were made. 
In fact, we consider the perturbed Euler type half-linear differential equation

$$
\left(\left(r_{1}(t)+\frac{r_{2}(t)}{\log ^{2} t}\right)^{1-p} \Phi\left(x^{\prime}\right)\right)^{\prime}+\left(s_{1}(t)+\frac{s_{2}(t)}{\log ^{2} t}\right) \frac{\Phi(x)}{t^{p}}=0, \quad t \in[\mathrm{e}, \infty),
$$

where $r_{i}, s_{i}:[\mathrm{e}, \infty) \rightarrow \mathbb{R}, i \in\{1,2\}$, are continuous functions such that

$$
r_{1}(t)+\frac{r_{2}(t)}{\log ^{2} t}>0, \quad t \in[\mathrm{e}, \infty)
$$

Henceforth, all equations will be considered for $t \in[\mathrm{e}, \infty)$. We assume that, for some $\alpha \in$ $[0,1)$, the inequalities

$$
\limsup _{t \rightarrow \infty} \frac{1}{t^{\alpha}} \int_{t}^{t+t^{\alpha}}\left|r_{i}(\tau)\right| d \tau<\infty, \quad \limsup _{t \rightarrow \infty} \frac{1}{t^{\alpha}} \int_{t}^{t+t^{\alpha}}\left|s_{i}(\tau)\right| d \tau<\infty, \quad i \in\{1,2\},
$$

hold. Thus, for $i \in\{1,2\}$, we can put

$$
A_{i}:=\sup _{t \geq \mathrm{e}} \frac{1}{t^{\alpha}} \int_{t}^{t+t^{\alpha}}\left|r_{i}(\tau)\right| d \tau<\infty, \quad B_{i}:=\sup _{t \geq \mathrm{e}} \frac{1}{t^{\alpha}} \int_{t}^{t+t^{\alpha}}\left|s_{i}(\tau)\right| d \tau<\infty .
$$

In addition, let there exist numbers $R_{1}, S_{1}>0$ and a continuous function $f:[\mathrm{e}, \infty) \rightarrow \mathbb{R}$ satisfying $\lim _{t \rightarrow \infty} f(t)=0$ and

$$
\left|R_{1}-\frac{1}{t^{\alpha}} \int_{t}^{t+t^{\alpha}} r_{1}(\tau) d \tau\right| \leq \frac{f(t)}{\log ^{2} t}, \quad\left|S_{1}-\frac{1}{t^{\alpha}} \int_{t}^{t+t^{\alpha}} s_{1}(\tau) d \tau\right| \leq \frac{f(t)}{\log ^{2} t}
$$

for all $t \in[\mathrm{e}, \infty)$.

The main result of this paper reads as follows.

Theorem 1.1 Let $R_{1}^{p-1} S_{1}=q^{-p}\left(\right.$ see (1.6)) and $R_{2}, S_{2} \in \mathbb{R}$.

(a) If

$$
\frac{1}{t^{\alpha}} \int_{t}^{t+t^{\alpha}} r_{2}(\tau) d \tau \geq R_{2}, \quad \frac{1}{t^{\alpha}} \int_{t}^{t+t^{\alpha}} s_{2}(\tau) d \tau \geq S_{2}
$$

for all large $t$ and

$$
q \frac{S_{2}}{S_{1}}+p \frac{R_{2}}{R_{1}}>\frac{q^{2}}{2},
$$

then Eq. (1.2) is oscillatory.

(b) If

$$
\frac{1}{t^{\alpha}} \int_{t}^{t+t^{\alpha}} r_{2}(\tau) d \tau \leq R_{2}, \quad \frac{1}{t^{\alpha}} \int_{t}^{t+t^{\alpha}} s_{2}(\tau) d \tau \leq S_{2}
$$

for all large $t$ and

$$
q \frac{S_{2}}{S_{1}}+p \frac{R_{2}}{R_{1}}<\frac{q^{2}}{2},
$$

then Eq. (1.2) is non-oscillatory. 


\section{Conditionally oscillatory half-linear equations: an overview}

The designation half-linear illustrates the fact that the solution space of Eq. (1.1) is homogeneous but it is not additive. This name was introduced in [2]. Of course, the lack of additivity is one of the main reasons of the fact that some tools widely used in the theory of linear equations are not available for half-linear equations. We can mention, e.g., the Wronskian identity (see [20]) or the Fredholm alternative (see [19]). On the bright side, many results from the theory of linear equations have been extended which gave birth to new advanced methods and approaches. At this place, we refer to the books $[1,16]$ that contain extensive theoretical background for half-linear equations.

The half-linear equations are situated between linear and non-linear equations on one side and between ordinary differential equations and partial differential equations on the other side. The first point of view is given by the fact that, putting $p=2$, we obtain linear equations. At this place, we should point out that results proved in this paper are not generalizations from linear equations to half-linear ones, but the presented research is new in the linear case as well. Of course, we prove all statements for general $p>1$ and one can obtain the linear versions of our results simply by considering $p=2$. Further, results about Eq. (1.1) are often first steps to extensive research in the field of non-linear equations, in particular with functions that preserve their sign in the place of function $\Phi$ (see, e.g., [18, $54,58])$. The second point of view comes directly from the relation of half-linear equations and (elliptic) partial differential equations with $p$-Laplacian. Since the function $\Phi$ is a one dimensional $p$-Laplacian, half-linear equations are scalar PDE's with $p$-Laplacian and one can use results obtained for scalar equations to deduce criteria for more general PDE's, especially concerning the so-called weak oscillation (see, e.g., [15, 28, 49]).

We recall that any non-trivial solution of a half-linear equation is said to be nonoscillatory if the solution is eventually positive or negative. Otherwise, the solution is called oscillatory. Since the Sturm separation theorem is extendable verbatim for halflinear equations, (non-)oscillation of one solution implies (non-)oscillation of all (nonzero) solutions of the given equation. Hence, half-linear equations can be categorized as oscillatory equations (if all solutions are oscillatory) and non-oscillatory equations (if all non-trivial solutions are non-oscillatory). For the half-linear version of the Sturm separation theorem, we refer again to $[1,16]$.

In the case a half-linear equation is non-oscillatory, the so-called principal and nonprincipal solutions can be introduced, similarly to the linear case. The asymptotic properties of these solutions play an important role in describing the asymptotics of any other solution of the equation; further, they can be an important tool in solving boundary value problems on non-compact intervals (see, e.g., [7-9]), because they can play the role of lower (upper) solutions for some modified problems. In particular, to obtain effective criteria for the solvability of the given BVP, various testing half-linear equations (those principal solutions are known) are used (see, e.g., the classic Euler equation or the RiemannWeber equation in [7] or the generalized Euler equation in [8]).

Conditionally oscillatory equations are ideal testing equations. Once we have a general conditionally oscillatory equation, we are able to obtain many oscillation or nonoscillation results by simply applying comparison theorems.

For completeness, we recall the definition of conditional oscillation. 
Definition 1 The equation

$$
\left(r(t) \Phi\left(x^{\prime}\right)\right)^{\prime}+\gamma s(t) \Phi(x)=0, \quad \gamma \in \mathbb{R}
$$

is said to be conditionally oscillatory if there exists the so-called critical oscillation constant $\Gamma>0$ with the property that Eq. (2.1) is oscillatory for $\gamma>\Gamma$ and non-oscillatory for $\gamma<\Gamma$.

The critical oscillation constant (obviously) depends on the functions $r$, s. We emphasize that the critical case $\gamma=\Gamma$ is not contained in the above definition. For some simple equations (with constant or periodic coefficients), it has been proved that non-oscillation is "more probable" in the critical case. Nevertheless, it appears to be challenging to decide the critical case for more general equations.

Now, we mention the most important milestones in the theory of conditionally oscillatory Euler type linear and half-linear differential equations. As far as we were able to find, the very first result of this kind comes from [43], where the linear equation

$$
x^{\prime \prime}+\frac{\gamma}{t^{2}} x=0
$$

has been proved to be conditionally oscillatory with critical oscillation constant $\Gamma=1 / 4$. Since the equation can be explicitly solved and its general solution is $c_{1} \sqrt{x}+c_{2} \sqrt{x} \log x$, $c_{1}, c_{2} \in \mathbb{R}$, in the critical case, we can directly see that Eq. (2.2) is non-oscillatory for $\gamma=$ $1 / 4$.

Next step has been made in $[25,52]$ one century later. There it was proved that conditional oscillation is preserved if we replace constant coefficients by periodic ones. Considering the equation

$$
\left(r(t) x^{\prime}\right)^{\prime}+\gamma \frac{s(t)}{t^{2}} x=0
$$

where $r, s$ are positive $\alpha$-periodic continuous functions, the critical oscillation constant is

$$
\Gamma=\frac{\alpha^{2}}{4}\left(\int_{0}^{\alpha} \frac{d \tau}{r(\tau)}\right)^{-1}\left(\int_{0}^{\alpha} s(\tau) d \tau\right)^{-1}
$$

This result is important also because of its continuation. We highlight at least [44-47], where several generalizations of this result are proved, and [53], where one can find the proof of the fact that Eq. (2.3) is non-oscillatory in the critical case.

Now, let us move to the half-linear equations. The basic result corresponding to Eq. (2.2) comes from [21,22]; the equation and the critical oscillation constant are

$$
\left(\Phi\left(x^{\prime}\right)\right)^{\prime}+\frac{\gamma}{t^{p}} \Phi(x)=0 \quad \text { and } \quad \Gamma=\left(\frac{p-1}{p}\right)^{p}
$$

Research in the field of half-linear equations continues. We mention, e.g., papers [13, $23,42,61]$. As our motivation and for the purpose of comparison and the reader's convenience, we formulate explicitly the main results of papers [32,39]. We emphasize that these papers have one important thing in common-the critical case is solved there. 
For the clarity of the formulation below, we introduce the number $q$ as the conjugate number with $p$, i.e., $p+q=p q$, and we mention the definition of the mean value for continuous functions.

Definition 2 Let a continuous function $f:[T, \infty) \rightarrow \mathbb{R}$ be such that the limit

$$
\bar{f}:=\lim _{t \rightarrow \infty} \frac{1}{t} \int_{a}^{a+t} f(s) d s
$$

is finite and exists uniformly with respect to $a \in[T, \infty)$. The number $\bar{f}$ is called the mean value of $f$.

Clearly, the mean value of any $\alpha$-periodic function $f: \mathbb{R} \rightarrow \mathbb{R}$ is

$$
\bar{f}=\frac{1}{\alpha} \int_{a}^{a+\alpha} f(s) d s,
$$

where $a$ is arbitrary. If we consider the sum of any periodic continuous functions $f_{i}$ for $i \in\{1, \ldots, n\}$, we immediately see that

$$
\overline{\sum_{i=1}^{n} f_{i}}=\sum_{i=1}^{n} \overline{f_{i}}
$$

In the first of the mentioned papers, i.e., in [32], the authors studied the equation

$$
\left(r^{1-p}(t) \Phi\left(x^{\prime}\right)\right)^{\prime}+\frac{s(t)}{t^{p}} \Phi(x)=0
$$

where $r, s:[\mathrm{e}, \infty) \rightarrow \mathbb{R}$ are continuous functions and $r$ is positive. The main result of [32] is as follows.

Theorem 2.1 If $r$ is $\alpha$-periodic with mean value $\bar{r}=1$ and $s$ is $\widehat{\alpha}$-periodic with mean value $\bar{s}=q^{-p}$, then Eq. (2.4) is non-oscillatory.

In fact, Theorem 2.1 is a continuation of certain previous results. Thus, for the reader's convenience, we mention the combination of Theorem 2.1 with [28, Theorem 9] which gives the next result (see directly [32, Theorem 4.4]).

Theorem 2.2 Let $r$ be $\alpha$-periodic, positive, and continuous, let s be $\widehat{\alpha}$-periodic and continuous for arbitrary $\alpha, \widehat{\alpha}>0$. Let us denote

$$
\Gamma:=q^{-p} \bar{r}^{1-p}=q^{-p}\left(\frac{1}{\alpha} \int_{0}^{\alpha} r(\tau) d \tau\right)^{1-p} .
$$

(a) If $\bar{s}>\Gamma$, then Eq. (2.4) is oscillatory.

(b) If $\bar{s} \leq \Gamma$, then Eq. (2.4) is non-oscillatory.

We add that Theorem 2.1 has been extended for the finite sums of periodic continuous functions as coefficients in [33]. Hence, the corresponding generalization of Theorem 2.2 is valid as well (it suffices to use again [28, Theorem 9]). 
Now, we focus on paper [39], where the equation

$$
\left(t^{\beta}\left(\sum_{i=1}^{m} R_{i}(t)\right)^{-1} x^{\prime}(t)\right)^{\prime}+t^{\beta-2} \sum_{j=1}^{n} S_{j}(t) x(t)=0, \quad \beta \in \mathbb{R} \backslash\{1\},
$$

is analyzed for arbitrary continuous periodic functions $R_{i}, i \in\{1, \ldots, m\}, S_{j}, j \in\{1, \ldots, n\}$, satisfying

$$
\inf _{t \geq 1} \sum_{i=1}^{m} R_{i}(t)>0 .
$$

The following theorem is proved in [39]. Note that this theorem coincides with Theorem 2.1 for $\beta=0$ and $p=2$.

Theorem 2.3 Consider Eq. (2.5). If

$$
\sum_{i=1}^{m} \overline{R_{i}} \sum_{j=1}^{n} \overline{S_{j}}=\frac{(\beta-1)^{2}}{4}
$$

then Eq. (2.5) is non-oscillatory.

The main goal of this paper is to describe the non-oscillation of Eq. (2.4) for more general coefficients $r, s$ than the periodic ones (or sums of periodic functions) in the critical case. This problem is covered by Theorem 1.1.

At the end of this section, we only briefly mention that the conditional oscillation of Euler type linear and half-linear equations is studied in the discrete case and in the case of dynamic equations on time scales as well. The results about difference equations are published, e.g., in $[11,36,41,63]$ and we refer to $[40,50,51,62]$ for results concerning dynamic equations on time scales. We will describe the research directed towards the discrete case and dynamic equations on time scales at the end of this paper within the collected open problems.

\section{Riccati and Prüfer technique}

In this section, we prepare needed tools which are the Riccati and Prüfer techniques and their combination. At first, we describe the half-linear Riccati transformation. We consider Eq. (2.4). We emphasize that the positivity of $r$ implies that the power of coefficient $r$ in the first term is not a restriction and it is considered from technical reasons in research papers. For a non-trivial solution $x$ of Eq. (2.4), we apply the transformation

$$
w(t)=r^{1-p}(t) \Phi\left(\frac{x^{\prime}(t)}{x(t)}\right)
$$

to get the so-called Riccati equation

$$
w^{\prime}(t)+\frac{s(t)}{t^{p}}+(p-1) r(t)|w(t)|^{q}=0
$$

associated to Eq. (2.4). Indeed, a solution $w$ of Eq. (3.1) exists whenever $x(t) \neq 0$. Therefore, the existence of the solution $w$ of Eq. (3.1) for $t \in[b, \infty)$ for some $b \geq$ e guarantees the 
non-oscillation of solution $x$ and the non-oscillation of one solution ensures (via the wellknown half-linear Sturm separation theorem) that Eq. (2.4) is non-oscillatory. The Riccati equation (3.1) is not sufficient for our purposes. Therefore, we apply another transformation

$$
v(t)=t^{p-1} w(t)
$$

to obtain the so-called adapted (or weighted) Riccati equation

$$
v^{\prime}(t)=\frac{p-1}{t}\left(v(t)-\frac{s(t)}{p-1}-r(t)|v(t)|^{q}\right) .
$$

For the description of the Prüfer technique, we should recall the notion of half-linear trigonometric functions. More precisely, we will need the half-linear sine and cosine functions denoted by $\sin _{p}$ and $\cos _{p}$, respectively. By the usual definition, $\sin _{p}$ is the odd periodic extension of the solution of the initial problem

$$
\left(\Phi\left(x^{\prime}\right)\right)^{\prime}+(p-1) \Phi(x)=0, \quad x(0)=0, x^{\prime}(0)=1
$$

and the half-linear cosine function $\cos _{p}$ is defined as the derivative of $\sin _{p}$. The period of $\sin _{p}$ and $\cos _{p}$ is

$$
2 \pi_{p}=\frac{4}{p} B\left(\frac{1}{p}, \frac{1}{q}\right)=\frac{4 \Gamma\left(\frac{1}{p}\right) \Gamma\left(\frac{1}{q}\right)}{p \Gamma\left(\frac{1}{p}+\frac{1}{q}\right)}=\frac{4 \pi}{p \sin \frac{\pi}{p}},
$$

where

$$
B(x, y)=\int_{0}^{1} \tau^{x-1}(1-\tau)^{y-1} d \tau, \quad x, y>0, \quad \Gamma(x)=\int_{0}^{\infty} \tau^{x-1} \mathrm{e}^{-\tau} d \tau, \quad x>0,
$$

are the standard Euler beta and gamma functions, respectively.

Regarding properties of $\sin _{p}$ and $\cos _{p}$, we mention only those which are used in this paper. For other properties and more details, we refer to [16] and the references cited therein. We recall the half-linear Pythagorean identity

$$
\left|\sin _{p} y\right|^{p}+\left|\cos _{p} y\right|^{p}=1, \quad y \in \mathbb{R}
$$

which also implies

$$
\left|\cos _{p} y\right|^{p} \leq 1, \quad\left|\Phi\left(\cos _{p} y\right) \sin _{p} y\right| \leq 1, \quad\left|\sin _{p} y\right|^{p} \leq 1, \quad y \in \mathbb{R} .
$$

Further, the continuous differentiability and periodicity of functions $\sin _{p}, \Phi\left(\sin _{p}\right), \Phi\left(\cos _{p}\right)$ provide the existence of positive numbers $L_{1}, L_{2}, L_{3}$ such that

$$
\begin{aligned}
& \left.|| \sin _{p} y\right|^{p}-\left|\sin _{p} z\right|^{p}|=| \Phi\left(\sin _{p} y\right) \sin _{p} y-\Phi\left(\sin _{p} z\right) \sin _{p} z\left|\leq L_{1}\right| y-z \mid, \quad y, z \in \mathbb{R}, \\
& \left.|| \cos _{p} y\right|^{p}-\left|\cos _{p} z\right|^{p}|=|\left|\Phi\left(\cos _{p} y\right)\right|^{\frac{p}{p-1}}-\left|\Phi\left(\cos _{p} z\right)\right|^{\frac{p}{p-1}}\left|\leq L_{2}\right| y-z \mid, \quad y, z \in \mathbb{R},
\end{aligned}
$$


and

$$
\left|\Phi\left(\cos _{p} y\right) \sin _{p} y-\Phi\left(\cos _{p} z\right) \sin _{p} z\right| \leq L_{3}|y-z|, \quad y, z \in \mathbb{R}
$$

Finally, we can proceed to the Prüfer technique and to the derivation of the equation for the Prüfer angle of Eq. (2.4). To do this, we use the modified half-linear Prüfer transformation

$$
x(t)=\rho(t) \sin _{p} \varphi(t), \quad x^{\prime}(t)=\frac{r(t) \rho(t)}{t} \cos _{p} \varphi(t) .
$$

Applying this transformation to (3.2), we obtain

$$
v(t)=\Phi\left(\frac{\cos _{p} \varphi(t)}{\sin _{p} \varphi(t)}\right)
$$

Now, we take into the account the fact that $\sin _{p}$ solves the equation in (3.4) to get

$$
\nu^{\prime}(t)=(1-p)\left(1+\left|\frac{\cos _{p} \varphi(t)}{\sin _{p} \varphi(t)}\right|^{p}\right) \varphi^{\prime}(t) .
$$

Next, we use (3.3) and (3.11) and, due to (3.10), we obtain

$$
\begin{aligned}
& (1-p)\left(1+\left|\frac{\cos _{p} \varphi(t)}{\sin _{p} \varphi(t)}\right|^{p}\right) \varphi^{\prime}(t) \\
& \quad=\frac{p-1}{t}\left(\Phi\left(\frac{\cos _{p} \varphi(t)}{\sin _{p} \varphi(t)}\right)-\frac{s(t)}{p-1}-r(t)\left|\frac{\cos _{p} \varphi(t)}{\sin _{p} \varphi(t)}\right|^{p}\right) .
\end{aligned}
$$

Applying the Pythagorean identity (3.5), we convert (3.12) to the final form of the equation for the Prüfer angle

$$
\varphi^{\prime}(t)=\frac{1}{t}\left(r(t)\left|\cos _{p} \varphi(t)\right|^{p}-\Phi\left(\cos _{p} \varphi(t)\right) \sin _{p} \varphi(t)+s(t) \frac{\left|\sin _{p} \varphi(t)\right|^{p}}{p-1}\right),
$$

which will be substantial in our method. The main idea is the equivalence of the boundedness of $\varphi$ and the non-oscillation of Eq. (2.4) (see directly [17, Corollary 4.1] or, e.g., [12, $45,53]$ ). Regarding a solution $\varphi$ of Eq. (3.13) (consider the right-hand side of Eq. (3.13) for values $\varphi(t)$ satisfying $\left.\sin _{p} \varphi(t)=0\right)$, it is seen that the set of all values $\varphi(t)$ is unbounded if and only if $\lim _{t \rightarrow \infty} \varphi(t)=\infty$.

\section{Oscillation and non-oscillation results}

Let us consider real numbers $a_{1}>0, b_{1}>0, a_{2}, b_{2}$. We use the equation

$$
\left(\left(a_{1}+\frac{a_{2}}{\log ^{2} t}\right)^{1-p} \Phi\left(x^{\prime}\right)\right)^{\prime}+\left(b_{1}+\frac{b_{2}}{\log ^{2} t}\right) \frac{\Phi(x)}{t^{p}}=0
$$


to study Eq. (1.2). The equation for the Prüfer angle associated to Eq. (4.1) has the form (see Eq. (3.13))

$$
\begin{aligned}
\varphi^{\prime}(t)= & \frac{1}{t}\left(\left(a_{1}+\frac{a_{2}}{\log ^{2} t}\right)\left|\cos _{p} \varphi(t)\right|^{p}-\Phi\left(\cos _{p} \varphi(t)\right) \sin _{p} \varphi(t)\right. \\
& \left.+\left(b_{1}+\frac{b_{2}}{\log ^{2} t}\right) \frac{\left|\sin _{p} \varphi(t)\right|^{p}}{p-1}\right)
\end{aligned}
$$

and the equation for the Prüfer angle associated to Eq. (1.2) takes the form

$$
\begin{aligned}
\varphi^{\prime}(t)= & \frac{1}{t}\left(\left(r_{1}(t)+\frac{r_{2}(t)}{\log ^{2} t}\right)\left|\cos _{p} \varphi(t)\right|^{p}-\Phi\left(\cos _{p} \varphi(t)\right) \sin _{p} \varphi(t)\right. \\
& \left.+\left(s_{1}(t)+\frac{s_{2}(t)}{\log ^{2} t}\right) \frac{\left|\sin _{p} \varphi(t)\right|^{p}}{p-1}\right) .
\end{aligned}
$$

Clearly, Eq. (4.2) is a special case of Eq. (4.3).

To prove Lemma 4.1 below, we need the well-known Sturm half-linear comparison theorem (which follows) and one known result in a special form (see Theorem 4.2 below).

Theorem 4.1 Let $c_{1}, c_{2}, d_{1}, d_{2}$ be continuous functions such that $c_{1}(t) \geq c_{2}(t)>0$ and $d_{2}(t) \geq d_{1}(t)$ for all large $t$. Let us consider the equations

$$
\begin{aligned}
& \left(c_{1}(t) \Phi\left(x^{\prime}\right)\right)^{\prime}+d_{1}(t) \Phi(x)=0 \\
& \left(c_{2}(t) \Phi\left(x^{\prime}\right)\right)^{\prime}+d_{2}(t) \Phi(x)=0 .
\end{aligned}
$$

(a) If Eq. (4.5) is non-oscillatory, then Eq. (4.4) is non-oscillatory as well.

(b) If Eq. (4.4) is oscillatory, then Eq. (4.5) is oscillatory as well.

Proof See, e.g., [16, Theorem 1.2.4].

Theorem 4.2 Let $a_{1}^{p-1} b_{1}=q^{-p}$.

(a) If

$$
q \frac{b_{2}}{b_{1}}+p \frac{a_{2}}{a_{1}}>\frac{q^{2}}{2}
$$

then Eq. (4.1) is oscillatory.

(b) If

$$
q \frac{b_{2}}{b_{1}}+p \frac{a_{2}}{a_{1}}<\frac{q^{2}}{2}
$$

then Eq. (4.1) is non-oscillatory.

Proof The theorem follows from results in each one of papers [10, 12, 17, 37].

We will also need the following auxiliary result. 
Lemma 4.1 Let $\varphi$ be a solution of Eq. (4.3) on some interval $[T, \infty)$, where $T \geq \mathrm{e}$, and let the function $\varphi_{\alpha}:[T, \infty) \rightarrow \mathbb{R}$ be defined by

$$
\varphi_{\alpha}(t):=\frac{1}{t^{\alpha}} \int_{t}^{t+t^{\alpha}} \varphi(\tau) d \tau, \quad t \in[T, \infty) .
$$

Then $\varphi_{\alpha}$ is a solution of the equation

$$
\begin{aligned}
\varphi_{\alpha}^{\prime}(t)= & \frac{1}{t}\left(\left(R_{1}+\frac{1}{\log ^{2} t}\left(\frac{1}{t^{\alpha}} \int_{t}^{t+t^{\alpha}} r_{2}(\tau) d \tau\right)\right)\left|\cos _{p} \varphi_{\alpha}(t)\right|^{p}\right. \\
& -\Phi\left(\cos _{p} \varphi_{\alpha}(t)\right) \sin _{p} \varphi_{\alpha}(t) \\
& \left.+\left(S_{1}+\frac{1}{\log ^{2} t}\left(\frac{1}{t^{\alpha}} \int_{t}^{t+t^{\alpha}} s_{2}(\tau) d \tau\right)\right) \frac{\left|\sin _{p} \varphi_{\alpha}(t)\right|^{p}}{p-1}+\frac{g(t)}{\log ^{2} t}\right)
\end{aligned}
$$

on $(T, \infty)$ for a continuous function $g:(T, \infty) \rightarrow \mathbb{R}$ satisfying $\lim _{t \rightarrow \infty} g(t)=0$.

Proof We express $\varphi_{\alpha}^{\prime}(t)$ for an arbitrarily given $t>T$. If $\alpha=0$, then

$$
\varphi_{\alpha}^{\prime}(t)=\left(\int_{t}^{t+1} \varphi(\tau) d \tau\right)^{\prime}=\varphi(t+1)-\varphi(t)=\int_{t}^{t+1} \varphi^{\prime}(\tau) d \tau=\frac{1}{t^{\alpha}} \int_{t}^{t+t^{\alpha}} \varphi^{\prime}(\tau) d \tau .
$$

If $\alpha \in(0,1)$, then

$$
\begin{aligned}
\varphi_{\alpha}^{\prime}(t) & =\left(\frac{1}{t^{\alpha}} \int_{t}^{t+t^{\alpha}} \varphi(\tau) d \tau\right)^{\prime} \\
& =-\frac{\alpha}{t^{\alpha+1}} \int_{t}^{t+t^{\alpha}} \varphi(\tau) d \tau+\frac{1}{t^{\alpha}}\left(\varphi\left(t+t^{\alpha}\right)\left(1+\frac{\alpha}{t^{1-\alpha}}\right)-\varphi(t)\right) \\
& =-\frac{\alpha}{t^{\alpha+1}} \int_{t}^{t+t^{\alpha}} \varphi(\tau) d \tau+\frac{\alpha}{t} \varphi\left(t+t^{\alpha}\right)+\frac{1}{t^{\alpha}} \int_{t}^{t+t^{\alpha}} \varphi^{\prime}(\tau) d \tau
\end{aligned}
$$

Now, we prove that

$$
\left|\varphi\left(t+t^{\alpha}\right)-\frac{1}{t^{\alpha}} \int_{t}^{t+t^{\alpha}} \varphi(\tau) d \tau\right|<\frac{h(t)}{\log ^{2} t}
$$

for all $t \geq T$ and for some continuous function $h:[T, \infty) \rightarrow \mathbb{R}$ such that $\lim _{t \rightarrow \infty} h(t)=0$. To obtain (4.10), we show that the inequality

$$
\left|\varphi(t+s)-\varphi_{\alpha}(t)\right| \leq \frac{A}{t^{1-\alpha}}
$$

holds for all $t \geq T, s \in\left[0, t^{\alpha}\right]$ and for some $A>0$. Of course, (4.11) gives (4.10) for $s=t^{\alpha}$. For any $t \geq T$, there exists $\sigma(t) \in\left[t, t+t^{\alpha}\right]$ such that $\varphi_{\alpha}(t)=\varphi(\sigma(t))$ (consider (4.6)). Hence, for any $t \geq T$, we obtain (see Eq. (4.3) together with (1.5) and (3.6))

$$
\begin{aligned}
& \left|\varphi(t+s)-\varphi_{\alpha}(t)\right| \\
& \quad=|\varphi(t+s)-\varphi(\sigma(t))|=\left|\int_{\sigma(t)}^{t+s} \varphi^{\prime}(\tau) d \tau\right| \leq \int_{t}^{t+t^{\alpha}}\left|\varphi^{\prime}(\tau)\right| d \tau
\end{aligned}
$$




$$
\begin{aligned}
= & \int_{t}^{t+t^{\alpha}} \mid \frac{1}{\tau}\left(\left(r_{1}(\tau)+\frac{r_{2}(\tau)}{\log ^{2} \tau}\right)\left|\cos _{p} \varphi(\tau)\right|^{p}-\Phi\left(\cos _{p} \varphi(\tau)\right) \sin _{p} \varphi(\tau)\right. \\
& \left.+\left(s_{1}(\tau)+\frac{s_{1}(\tau)}{\log ^{2} \tau}\right) \frac{\left|\sin _{p} \varphi(\tau)\right|^{p}}{p-1}\right) \mid d \tau \\
\leq & \frac{1}{t^{1-\alpha}}\left(\frac{1}{t^{\alpha}} \int_{t}^{t+t^{\alpha}}\left(\left|r_{1}(\tau)\right|+\frac{\left|r_{2}(\tau)\right|}{\log ^{2} \mathrm{e}}\right)\left|\cos _{p} \varphi(\tau)\right|^{p} d \tau\right. \\
& +\frac{1}{t^{\alpha}} \int_{t}^{t+t^{\alpha}}\left|\Phi\left(\cos _{p} \varphi(\tau)\right) \sin _{p} \varphi(\tau)\right| d \tau \\
& \left.+\frac{1}{t^{\alpha}} \int_{t}^{t+t^{\alpha}}\left(\left|s_{1}(\tau)\right|+\frac{\left|s_{2}(\tau)\right|}{\log ^{2} \mathrm{e}}\right) \frac{\left|\sin _{p} \varphi(\tau)\right|^{p}}{p-1} d \tau\right) \\
\leq & \frac{1}{t^{1-\alpha}}\left(\frac{1}{t^{\alpha}} \int_{t}^{t+t^{\alpha}}\left(\left|r_{1}(\tau)\right|+\left|r_{2}(\tau)\right|+1+\left(\left|s_{1}(\tau)\right|+\left|s_{2}(\tau)\right|\right) \frac{1}{p-1}\right) d \tau\right) \\
\leq & \frac{1}{t^{1-\alpha}}\left(A_{1}+A_{2}+1+\frac{B_{1}+B_{2}}{p-1}\right),
\end{aligned}
$$

i.e., (4.11) is valid for

$$
A:=A_{1}+A_{2}+1+\frac{B_{1}+B_{2}}{p-1} .
$$

Considering (4.8), (4.9), and (4.10), we find that the inequality

$$
\left|\varphi_{\alpha}^{\prime}(t)-\frac{1}{t^{\alpha}} \int_{t}^{t+t^{\alpha}} \varphi^{\prime}(\tau) d \tau\right|<\frac{h(t)}{t \log ^{2} t}
$$

holds for all $t \geq T$ and for a continuous function $h:[T, \infty) \rightarrow \mathbb{R}$ such that $\lim _{t \rightarrow \infty} h(t)=0$.

Thus, we consider (see again Eq. (4.3))

$$
\begin{aligned}
\frac{1}{t^{\alpha}} \int_{t}^{t+t^{\alpha}} \varphi^{\prime}(\tau) d \tau \\
=\frac{1}{t^{\alpha}} \int_{t}^{t+t^{\alpha}} \frac{1}{\tau}\left(\left(r_{1}(\tau)+\frac{r_{2}(\tau)}{\log ^{2} \tau}\right)\left|\cos _{p} \varphi(\tau)\right|^{p}\right. \\
\left.\quad-\Phi\left(\cos _{p} \varphi(\tau)\right) \sin _{p} \varphi(\tau)+\left(s_{1}(\tau)+\frac{s_{2}(\tau)}{\log ^{2} \tau}\right) \frac{\left|\sin _{p} \varphi(\tau)\right|^{p}}{p-1}\right) d \tau .
\end{aligned}
$$

Since (see (1.5) and (3.6))

$$
\begin{aligned}
& \mid \int_{t}^{t+t^{\alpha}} \frac{1}{\tau}\left(\left(r_{1}(\tau)+\frac{r_{2}(\tau)}{\log ^{2} \tau}\right)\left|\cos _{p} \varphi(\tau)\right|^{p}\right. \\
& \left.\quad-\Phi\left(\cos _{p} \varphi(\tau)\right) \sin _{p} \varphi(\tau)+\left(s_{1}(\tau)+\frac{s_{2}(\tau)}{\log ^{2} \tau}\right) \frac{\left|\sin _{p} \varphi(\tau)\right|^{p}}{p-1}\right) d \tau \\
& \quad-\int_{t}^{t+t^{\alpha}} \frac{1}{t}\left(\left(r_{1}(\tau)+\frac{r_{2}(\tau)}{\log ^{2} \tau}\right)\left|\cos _{p} \varphi(\tau)\right|^{p}\right. \\
& \left.\quad-\Phi\left(\cos _{p} \varphi(\tau)\right) \sin _{p} \varphi(\tau)+\left(s_{1}(\tau)+\frac{s_{2}(\tau)}{\log ^{2} \tau}\right) \frac{\left|\sin _{p} \varphi(\tau)\right|^{p}}{p-1}\right) d \tau \mid \\
& \leq \int_{t}^{t+t^{\alpha}}\left(\frac{1}{t}-\frac{1}{\tau}\right)\left(\left(\left|r_{1}(\tau)\right|+\frac{\left|r_{2}(\tau)\right|}{\log ^{2} \mathrm{e}}\right)+1+\left(\left|s_{1}(\tau)\right|+\frac{\left|s_{2}(\tau)\right|}{\log ^{2} \mathrm{e}}\right) \frac{1}{p-1}\right) d \tau
\end{aligned}
$$




$$
\leq t^{\alpha}\left(\frac{1}{t}-\frac{1}{t+t^{\alpha}}\right)\left(A_{1}+A_{2}+1+\frac{B_{1}+B_{2}}{p-1}\right) \leq \frac{A}{t^{2(1-\alpha)}}
$$

where $A$ is from (4.12), to prove the statement of the theorem, it suffices to obtain the inequalities

$$
\begin{aligned}
& \left.\left|\frac{1}{t^{\alpha}} \int_{t}^{t+t^{\alpha}} r_{1}(\tau)\right| \cos _{p} \varphi(\tau)\right|^{p} d \tau-R_{1}\left|\cos _{p} \varphi_{\alpha}(t)\right|^{p} \mid \leq \frac{F_{1}(t)}{\log ^{2} t} \\
& \left.\left|\frac{1}{t^{\alpha}} \int_{t}^{t+t^{\alpha}} \frac{r_{2}(\tau)}{\log ^{2} \tau}\right| \cos _{p} \varphi(\tau)\right|^{p} d \tau-\frac{\left|\cos _{p} \varphi_{\alpha}(t)\right|^{p}}{\log ^{2} t}\left(\frac{1}{t^{\alpha}} \int_{t}^{t+t^{\alpha}} r_{2}(\tau) d \tau\right) \mid \leq \frac{F_{2}(t)}{\log ^{2} t} \\
& \left|\frac{1}{t^{\alpha}} \int_{t}^{t+t^{\alpha}} \Phi\left(\cos _{p} \varphi(\tau)\right) \sin _{p} \varphi(\tau) d \tau-\Phi\left(\cos _{p} \varphi_{\alpha}(t)\right) \sin _{p} \varphi_{\alpha}(t)\right| \leq \frac{F_{3}(t)}{\log ^{2} t} \\
& \left|\frac{1}{t^{\alpha}} \int_{t}^{t+t^{\alpha}} s_{1}(\tau) \frac{\left|\sin _{p} \varphi(\tau)\right|^{p}}{p-1} d \tau-S_{1} \frac{\left|\sin _{p} \varphi_{\alpha}(t)\right|^{p}}{p-1}\right| \leq \frac{F_{4}(t)}{\log ^{2} t}
\end{aligned}
$$

and

$$
\left|\frac{1}{t^{\alpha}} \int_{t}^{t+t^{\alpha}} \frac{s_{2}(\tau)}{\log ^{2} \tau} \frac{\left|\sin _{p} \varphi(\tau)\right|^{p}}{p-1} d \tau-\frac{\left|\sin _{p} \varphi_{\alpha}(t)\right|^{p}}{(p-1) \log ^{2} t}\left(\frac{1}{t^{\alpha}} \int_{t}^{t+t^{\alpha}} s_{2}(\tau) d \tau\right)\right| \leq \frac{F_{5}(t)}{\log ^{2} t}
$$

for all $t \geq T$ and certain continuous functions $F_{1}, F_{2}, F_{3}, F_{4}, F_{5}:[T, \infty) \rightarrow \mathbb{R}$ satisfying $\lim _{t \rightarrow \infty} F_{i}(t)=0$ for each $i \in\{1,2,3,4,5\}$. Indeed (consider (4.13), (4.14), and (4.15)), inequalities (4.16)-(4.20) give (4.7).

We have (see (1.5), (1.6), (3.6), (3.8), and (4.11))

$$
\begin{aligned}
&\left.\left|\frac{1}{t^{\alpha}} \int_{t}^{t+t^{\alpha}} r_{1}(\tau)\right| \cos _{p} \varphi(\tau)\right|^{p} d \tau-R_{1}\left|\cos _{p} \varphi_{\alpha}(t)\right|^{p} \mid \\
& \leq\left.\left|\frac{1}{t^{\alpha}} \int_{t}^{t+t^{\alpha}} r_{1}(\tau)\right| \cos _{p} \varphi(\tau)\right|^{p} d \tau-\frac{1}{t^{\alpha}} \int_{t}^{t+t^{\alpha}} r_{1}(\tau) d \tau\left|\cos _{p} \varphi_{\alpha}(t)\right|^{p} \mid \\
& \quad+\left.\left|\frac{1}{t^{\alpha}} \int_{t}^{t+t^{\alpha}} r_{1}(\tau) d \tau\right| \cos _{p} \varphi_{\alpha}(t)\right|^{p}-R_{1}\left|\cos _{p} \varphi_{\alpha}(t)\right|^{p} \mid \\
& \leq\left.\frac{1}{t^{\alpha}} \int_{t}^{t+t^{\alpha}}\left|r_{1}(\tau)\right| \cdot|| \cos _{p} \varphi(\tau)\right|^{p}-\left|\cos _{p} \varphi_{\alpha}(t)\right|^{p} \mid d \tau \\
&+\left|\frac{1}{t^{\alpha}} \int_{t}^{t+t^{\alpha}} r_{1}(\tau) d \tau-R_{1}\right| \\
& \leq \frac{1}{t^{\alpha}} \int_{t}^{t+t^{\alpha}}\left|r_{1}(\tau)\right| L_{2}\left|\varphi(\tau)-\varphi_{\alpha}(t)\right| d \tau+\frac{f(t)}{\log ^{2} t} \\
& \leq \frac{L_{2} A}{t^{1-\alpha}}\left(\frac{1}{t^{\alpha}} \int_{t}^{t+t^{\alpha}}\left|r_{1}(\tau)\right| d \tau\right)+\frac{f(t)}{\log ^{2} t} \leq \frac{L_{2} A A_{1}}{t^{1-\alpha}}+\frac{f(t)}{\log ^{2} t}
\end{aligned}
$$

for all $t \geq T$ and for a continuous function $f:[T, \infty) \rightarrow \mathbb{R}$ such that $\lim _{t \rightarrow \infty} f(t)=0$. Thus, (4.16) is true.

To prove (4.17), we use the limit

$$
\lim _{t \rightarrow \infty} \log ^{3} t\left(\frac{1}{\log ^{2} t}-\frac{1}{\log ^{2}\left(t+t^{\alpha}\right)}\right)=0
$$


Došlá et al. Journal of Inequalities and Applications

(2019) 2019:189

Page 13 of 30

For all large $t$, we obtain (see (1.5), (3.6), (3.8), (4.11), and (4.22))

$$
\begin{aligned}
&\left.\left|\frac{1}{t^{\alpha}} \int_{t}^{t+t^{\alpha}} \frac{r_{2}(\tau)}{\log ^{2} \tau}\right| \cos _{p} \varphi(\tau)\right|^{p} d \tau-\frac{\left|\cos _{p} \varphi_{\alpha}(t)\right|^{p}}{\log ^{2} t}\left(\frac{1}{t^{\alpha}} \int_{t}^{t+t^{\alpha}} r_{2}(\tau) d \tau\right) \mid \\
&=\left.\left|\frac{1}{t^{\alpha}} \int_{t}^{t+t^{\alpha}} \frac{r_{2}(\tau)}{\log ^{2} t} d \tau\right| \cos _{p} \varphi_{\alpha}(t)\right|^{p}-\frac{1}{t^{\alpha}} \int_{t}^{t+t^{\alpha}} \frac{r_{2}(\tau)}{\log ^{2} \tau}\left|\cos _{p} \varphi(\tau)\right|^{p} d \tau \mid \\
& \leq\left.\left|\frac{1}{t^{\alpha}} \int_{t}^{t+t^{\alpha}} \frac{r_{2}(\tau)}{\log ^{2} t} d \tau\right| \cos _{p} \varphi_{\alpha}(t)\right|^{p}-\frac{1}{t^{\alpha}} \int_{t}^{t+t^{\alpha}} \frac{r_{2}(\tau)}{\log ^{2} \tau} d \tau\left|\cos _{p} \varphi_{\alpha}(t)\right|^{p} \mid \\
& \quad+\left.\left|\frac{1}{t^{\alpha}} \int_{t}^{t+t^{\alpha}} \frac{r_{2}(\tau)}{\log ^{2} \tau} d \tau\right| \cos _{p} \varphi_{\alpha}(t)\right|^{p}-\frac{1}{t^{\alpha}} \int_{t}^{t+t^{\alpha}} \frac{r_{2}(\tau)}{\log ^{2} \tau}\left|\cos _{p} \varphi(\tau)\right|^{p} d \tau \mid \\
& \leq \frac{1}{t^{\alpha}} \int_{t}^{t+t^{\alpha}}\left(\frac{1}{\log ^{2} t}-\frac{1}{\log ^{2}\left(t+t^{\alpha}\right)}\right)\left|r_{2}(\tau)\right| d \tau \\
& \quad+\left.\frac{1}{t^{\alpha} \log ^{2} \mathrm{e}} \int_{t}^{t+t^{\alpha}}\left|r_{2}(\tau)\right| \cdot|| \cos _{p} \varphi_{\alpha}(t)\right|^{p}-\left|\cos _{p} \varphi(\tau)\right|^{p} \mid d \tau \\
& \leq \frac{1}{\log ^{3} t}\left(\frac{1}{t^{\alpha}} \int_{t}^{t+t^{\alpha}}\left|r_{2}(\tau)\right| d \tau\right)+\frac{1}{t^{\alpha}} \int_{t}^{t+t^{\alpha}}\left|r_{2}(\tau)\right| L_{2}\left|\varphi_{\alpha}(t)-\varphi(\tau)\right| d \tau \\
& \leq \frac{A_{2}}{\log ^{3} t}+\frac{L_{2} A}{t^{1-\alpha}}\left(\frac{1}{t^{\alpha}} \int_{t}^{t+t^{\alpha}}\left|r_{2}(\tau)\right| d \tau\right) \leq \frac{A_{2}}{\log ^{3} t}+\frac{L_{2} A A_{2}}{t^{1-\alpha}},
\end{aligned}
$$

i.e., (4.17) is valid.

Furthermore, (see (3.9) and (4.11))

$$
\begin{aligned}
& \left|\Phi\left(\cos _{p} \varphi_{\alpha}(t)\right) \sin _{p} \varphi_{\alpha}(t)-\frac{1}{t^{\alpha}} \int_{t}^{t+t^{\alpha}} \Phi\left(\cos _{p} \varphi(\tau)\right) \sin _{p} \varphi(\tau) d \tau\right| \\
& \leq \frac{1}{t^{\alpha}} \int_{t}^{t+t^{\alpha}}\left|\Phi\left(\cos _{p} \varphi_{\alpha}(t)\right) \sin _{p} \varphi_{\alpha}(t)-\Phi\left(\cos _{p} \varphi(\tau)\right) \sin _{p} \varphi(\tau)\right| d \tau \\
& \quad \leq \frac{1}{t^{\alpha}} \int_{t}^{t+t^{\alpha}} L_{3}\left|\varphi_{\alpha}(t)-\varphi(\tau)\right| d \tau \leq \frac{1}{t^{\alpha}} \int_{t}^{t+t^{\alpha}} \frac{L_{3} A}{t^{1-\alpha}} d \tau=\frac{L_{3} A}{t^{1-\alpha}}
\end{aligned}
$$

i.e., (4.18) holds.

To obtain (4.19), we proceed analogously as in (4.21), where we have (see (1.5), (1.6), (3.6), (3.7), and (4.11))

$$
\begin{aligned}
& \left|\frac{1}{t^{\alpha}} \int_{t}^{t+t^{\alpha}} s_{1}(\tau) \frac{\left|\sin _{p} \varphi(\tau)\right|^{p}}{p-1} d \tau-S_{1} \frac{\left|\sin _{p} \varphi_{\alpha}(t)\right|^{p}}{p-1}\right| \\
& \leq\left|\frac{1}{t^{\alpha}} \int_{t}^{t+t^{\alpha}} s_{1}(\tau) \frac{\left|\sin _{p} \varphi(\tau)\right|^{p}}{p-1} d \tau-\frac{1}{t^{\alpha}} \int_{t}^{t+t^{\alpha}} s_{1}(\tau) d \tau \frac{\left|\sin _{p} \varphi_{\alpha}(t)\right|^{p}}{p-1}\right| \\
& \quad+\left|\frac{1}{t^{\alpha}} \int_{t}^{t+t^{\alpha}} s_{1}(\tau) d \tau \frac{\left|\sin _{p} \varphi_{\alpha}(t)\right|^{p}}{p-1}-S_{1} \frac{\left|\sin _{p} \varphi_{\alpha}(t)\right|^{p}}{p-1}\right| \\
& \leq \\
& \quad \frac{1}{p-1}\left(\left.\frac{1}{t^{\alpha}} \int_{t}^{t+t^{\alpha}}\left|s_{1}(\tau)\right| \cdot|| \sin _{p} \varphi(\tau)\right|^{p}-\left|\sin _{p} \varphi_{\alpha}(t)\right|^{p} \mid d \tau\right. \\
& \left.\quad+\left|\frac{1}{t^{\alpha}} \int_{t}^{t+t^{\alpha}} s_{1}(\tau) d \tau-S_{1}\right|\right)
\end{aligned}
$$




$$
\begin{aligned}
& \leq \frac{1}{p-1}\left(\frac{1}{t^{\alpha}} \int_{t}^{t+t^{\alpha}}\left|s_{1}(\tau)\right| L_{1}\left|\varphi(\tau)-\varphi_{\alpha}(t)\right| d \tau+\frac{f(t)}{\log ^{2} t}\right) \\
& \leq \frac{1}{p-1}\left(\frac{L_{1} A}{t^{1-\alpha}}\left(\frac{1}{t^{\alpha}} \int_{t}^{t+t^{\alpha}}\left|s_{1}(\tau)\right| d \tau\right)+\frac{f(t)}{\log ^{2} t}\right) \\
& \leq \frac{1}{p-1}\left(\frac{L_{1} A B_{1}}{t^{1-\alpha}}+\frac{f(t)}{\log ^{2} t}\right),
\end{aligned}
$$

where the function $f:[T, \infty) \rightarrow \mathbb{R}$ is continuous with $\lim _{t \rightarrow \infty} f(t)=0$.

To finish the proof, we prove (4.20) which follows from (see (1.5), (3.6), (3.7), (4.11), and

$$
\begin{aligned}
&\left|\frac{1}{t^{\alpha}} \int_{t}^{t+t^{\alpha}} \frac{s_{2}(\tau)}{\log ^{2} \tau} \frac{\left|\sin _{p} \varphi(\tau)\right|^{p}}{p-1} d \tau-\frac{\left|\sin _{p} \varphi_{\alpha}(t)\right|^{p}}{(p-1) \log ^{2} t}\left(\frac{1}{t^{\alpha}} \int_{t}^{t+t^{\alpha}} s_{2}(\tau) d \tau\right)\right| \\
&=\left|\frac{1}{t^{\alpha}} \int_{t}^{t+t^{\alpha}} \frac{s_{2}(\tau)}{\log ^{2} t} d \tau \frac{\left|\sin _{p} \varphi_{\alpha}(t)\right|^{p}}{p-1}-\frac{1}{t^{\alpha}} \int_{t}^{t+t^{\alpha}} \frac{s_{2}(\tau)}{\log ^{2} \tau} \frac{\left|\sin _{p} \varphi(\tau)\right|^{p}}{p-1} d \tau\right| \\
& \leq\left|\frac{1}{t^{\alpha}} \int_{t}^{t+t^{\alpha}} \frac{s_{2}(\tau)}{\log ^{2} t} d \tau \frac{\left|\sin _{p} \varphi_{\alpha}(t)\right|^{p}}{p-1}-\frac{1}{t^{\alpha}} \int_{t}^{t+t^{\alpha}} \frac{s_{2}(\tau)}{\log ^{2} \tau} d \tau \frac{\left|\sin _{p} \varphi_{\alpha}(t)\right|^{p}}{p-1}\right| \\
& \quad+\left|\frac{1}{t^{\alpha}} \int_{t}^{t+t^{\alpha}} \frac{s_{2}(\tau)}{\log ^{2} \tau} \frac{\left|\sin _{p} \varphi_{\alpha}(t)\right|^{p}}{p-1} d \tau-\frac{1}{t^{\alpha}} \int_{t}^{t+t^{\alpha}} \frac{s_{2}(\tau)}{\log ^{2} \tau} \frac{\left|\sin _{p} \varphi(\tau)\right|^{p}}{p-1} d \tau\right| \\
& \leq \frac{1}{p-1}\left(\frac{1}{t^{\alpha}} \int_{t}^{t+t^{\alpha}}\left(\frac{1}{\log ^{2} t}-\frac{1}{\log ^{2}\left(t+t^{\alpha}\right)}\right)\left|s_{2}(\tau)\right| d \tau\right. \\
&\left.+\left.\frac{1}{t^{\alpha} \log ^{2} \mathrm{e}} \int_{t}^{t+t^{\alpha}}\left|s_{2}(\tau)\right| \cdot|| \sin _{p} \varphi_{\alpha}(t)\right|^{p}-\left|\sin _{p} \varphi(\tau)\right|^{p} \mid d \tau\right) \\
& \leq \frac{1}{p-1}\left(\frac{1}{\log ^{3} t}\left(\frac{1}{t^{\alpha}} \int_{t}^{t+t^{\alpha}}\left|s_{2}(\tau)\right| d \tau\right)+\frac{1}{t^{\alpha}} \int_{t}^{t+t^{\alpha}}\left|s_{2}(\tau)\right| L_{1}\left|\varphi_{\alpha}(t)-\varphi(\tau)\right| d \tau\right) \\
& \leq \frac{1}{p-1}\left(\frac{B_{2}}{\log ^{3} t}+\frac{L_{1} A}{t^{1-\alpha}}\left(\frac{1}{t^{\alpha}} \int_{t}^{t+t^{\alpha}}\left|s_{2}(\tau)\right| d \tau\right)\right) \\
& \leq \frac{B_{2}}{p-1}\left(\frac{1}{\log ^{3} t}+\frac{L_{1} A}{t^{1-\alpha}}\right),
\end{aligned}
$$

where $t \geq T$ is sufficiently large.

Now, we can prove Theorem 1.1 as presented in the Introduction.

Proof of Theorem 1.1 It is a well-known fact (see, e.g., [12, 17, 45, 53]) that Eq. (1.2) is oscillatory if and only if the Prüfer angle (given by Eq. (4.3)) is unbounded from above. Further, from the proof of Lemma 4.1 (see (4.11)), we find that Eq. (1.2) is oscillatory if and only if $\limsup _{t \rightarrow \infty} \varphi_{\alpha}(t)=\infty$ for an arbitrary solution $\varphi$ of Eq. (4.3) on some interval $[T, \infty)$. In particular, Eq. (4.1) is non-oscillatory if and only if any solution of Eq. (4.2) is bounded from above.

In the first part of this proof, we prove that $\lim _{t \rightarrow \infty} \varphi_{\alpha}(t)=\infty$ if (1.7) and (1.8) are valid. Let $\delta>0$ be so small that (see (1.8))

$$
q \frac{S_{2}-\delta}{S_{1}}+p \frac{R_{2}-\delta}{R_{1}}>\frac{q^{2}}{2}
$$


For all large $t$, we have (see (1.7), (3.5), and (4.7))

$$
\begin{aligned}
\varphi_{\alpha}^{\prime}(t)= & \frac{1}{t}\left(\left(R_{1}+\frac{1}{\log ^{2} t}\left(\frac{1}{t^{\alpha}} \int_{t}^{t+t^{\alpha}} r_{2}(\tau) d \tau\right)\right)\left|\cos _{p} \varphi_{\alpha}(t)\right|^{p}\right. \\
& -\Phi\left(\cos _{p} \varphi_{\alpha}(t)\right) \sin _{p} \varphi_{\alpha}(t) \\
& \left.+\left(S_{1}+\frac{1}{\log ^{2} t}\left(\frac{1}{t^{\alpha}} \int_{t}^{t+t^{\alpha}} s_{2}(\tau) d \tau\right)\right) \frac{\left|\sin _{p} \varphi_{\alpha}(t)\right|^{p}}{p-1}+\frac{g(t)}{\log ^{2} t}\right) \\
\geq & \frac{1}{t}\left(\left(R_{1}+\frac{R_{2}-|g(t)|}{\log ^{2} t}\right)\left|\cos _{p} \varphi_{\alpha}(t)\right|^{p}-\Phi\left(\cos _{p} \varphi_{\alpha}(t)\right) \sin _{p} \varphi_{\alpha}(t)\right. \\
& \left.+\left(S_{1}+\frac{S_{2}-(p-1)|g(t)|}{\log ^{2} t}\right) \frac{\left|\sin _{p} \varphi_{\alpha}(t)\right|^{p}}{p-1}\right) \\
\geq & \frac{1}{t}\left(\left(R_{1}+\frac{R_{2}-\delta}{\log ^{2} t}\right)\left|\cos _{p} \varphi_{\alpha}(t)\right|^{p}-\Phi\left(\cos _{p} \varphi_{\alpha}(t)\right) \sin _{p} \varphi_{\alpha}(t)\right. \\
& \left.+\left(S_{1}+\frac{S_{2}-\delta}{\log ^{2} t}\right) \frac{\left|\sin _{p} \varphi_{\alpha}(t)\right|^{p}}{p-1}\right) .
\end{aligned}
$$

The equation

$$
\begin{aligned}
\psi^{\prime}(t)= & \frac{1}{t}\left(\left(R_{1}+\frac{R_{2}-\delta}{\log ^{2} t}\right)\left|\cos _{p} \psi(t)\right|^{p}-\Phi\left(\cos _{p} \psi(t)\right) \sin _{p} \psi(t)\right. \\
& \left.+\left(S_{1}+\frac{S_{2}-\delta}{\log ^{2} t}\right) \frac{\left|\sin _{p} \psi(t)\right|^{p}}{p-1}\right)
\end{aligned}
$$

has the form of Eq. (4.2). From (4.23) and Theorem 4.2, (a), we know that Eq. (4.25) has solutions that are unbounded (from above). Comparing (4.24) with Eq. (4.25), we obtain (see (4.11))

$$
\lim _{t \rightarrow \infty} \varphi_{\alpha}(t)=\lim _{t \rightarrow \infty} \varphi(t)=\infty
$$

i.e., Eq. (1.2) is oscillatory.

In the second part of the proof, we show that $\limsup _{t \rightarrow \infty} \varphi_{\alpha}(t)<\infty$ if (1.9) and (1.10) are valid. Let $\delta>0$ be such that (see (1.10))

$$
q \frac{S_{2}+\delta}{S_{1}}+p \frac{R_{2}+\delta}{R_{1}}<\frac{q^{2}}{2} .
$$

Analogously as in (4.24), for all large $t$, we have (see (1.9), (3.5), and (4.7))

$$
\begin{aligned}
\varphi_{\alpha}^{\prime}(t) \leq & \frac{1}{t}\left(\left(R_{1}+\frac{R_{2}+|g(t)|}{\log ^{2} t}\right)\left|\cos _{p} \varphi_{\alpha}(t)\right|^{p}-\Phi\left(\cos _{p} \varphi_{\alpha}(t)\right) \sin _{p} \varphi_{\alpha}(t)\right. \\
& \left.+\left(S_{1}+\frac{S_{2}+(p-1)|g(t)|}{\log ^{2} t}\right) \frac{\left|\sin _{p} \varphi_{\alpha}(t)\right|^{p}}{p-1}\right) \\
\leq & \frac{1}{t}\left(\left(R_{1}+\frac{R_{2}+\delta}{\log ^{2} t}\right)\left|\cos _{p} \varphi_{\alpha}(t)\right|^{p}-\Phi\left(\cos _{p} \varphi_{\alpha}(t)\right) \sin _{p} \varphi_{\alpha}(t)\right. \\
& \left.+\left(S_{1}+\frac{S_{2}+\delta}{\log ^{2} t}\right) \frac{\left|\sin _{p} \varphi_{\alpha}(t)\right|^{p}}{p-1}\right) .
\end{aligned}
$$


Now, we consider the equation

$$
\begin{aligned}
\psi^{\prime}(t)= & \frac{1}{t}\left(\left(R_{1}+\frac{R_{2}+\delta}{\log ^{2} t}\right)\left|\cos _{p} \psi(t)\right|^{p}-\Phi\left(\cos _{p} \psi(t)\right) \sin _{p} \psi(t)\right. \\
& \left.+\left(S_{1}+\frac{S_{2}+\delta}{\log ^{2} t}\right) \frac{\left|\sin _{p} \psi(t)\right|^{p}}{p-1}\right)
\end{aligned}
$$

which has the form of Eq. (4.2). Theorem 4.2, (b) (see (4.26)) guarantees that all solutions of Eq. (4.28) are bounded (from above). Considering (4.27) and Eq. (4.28), we have (see $(4.11))$

$$
\limsup _{t \rightarrow \infty} \varphi_{\alpha}(t)=\limsup _{t \rightarrow \infty} \varphi(t)<\infty
$$

which gives the non-oscillation of Eq. (1.2).

To show that Theorem 1.1 improves Theorem 2.1 mentioned in the Introduction as the basic motivation, we explicitly formulate the following result in full. In fact, Theorem 4.3 below is a trivial consequence of Theorem 1.1.

Theorem 4.3 Consider the equation

$$
\left(r(t) \Phi\left(x^{\prime}\right)\right)^{\prime}+\frac{s(t)}{t^{p}} \Phi(x)=0,
$$

where $r>0$, s are continuous functions such that there exist $\alpha \in[0,1), R, S>0$, and a continuous function $f:[\mathrm{e}, \infty) \rightarrow \mathbb{R}$ satisfying $\lim _{t \rightarrow \infty} f(t)=0$ for which

$$
\limsup _{t \rightarrow \infty} \frac{1}{t^{\alpha}} \int_{t}^{t+t^{\alpha}}|s(\tau)| d \tau<\infty
$$

and

$$
\begin{aligned}
& \left|R-\frac{1}{t^{\alpha}} \int_{t}^{t+t^{\alpha}} r^{\frac{1}{1-p}}(\tau) d \tau\right| \leq \frac{f(t)}{\log ^{2} t}, \quad t \in[\mathrm{e}, \infty), \\
& \left|S-\frac{1}{t^{\alpha}} \int_{t}^{t+t^{\alpha}} S(\tau) d \tau\right| \leq \frac{f(t)}{\log ^{2} t}, \quad t \in[\mathrm{e}, \infty) .
\end{aligned}
$$

If $R^{p-1} S=q^{-p}$, then Eq. (4.29) is non-oscillatory.

Proof The theorem is a corollary of Theorem 1.1. It suffices to use Theorem 1.1, (b) for Eq. (1.2) with $r_{2} \equiv 0, s_{2} \equiv 0$.

Remark 1 We repeat that Theorem 4.3 generalizes Theorem 2.1. Theorem 4.3 generalizes Theorem 2.3 for $\beta=0$ as well. It is easy to verify this fact using [39, Lemma 3.5] for the choice $\alpha=1 / 2$. Indeed, [39, Lemma 3.5] implies (4.31) and (4.32) for $r, s$ which are sums of continuous periodic functions. Note that (4.30) is trivially satisfied for any sum of continuous periodic functions in the position of $s$. We add that, applying the presented process, a generalization of Theorem 2.3 concerning a general $\beta$ is actually not possible to obtain because no result corresponding to Theorem 4.2 for $\beta \neq 0$ is known. We also refer to the open problem (IV) in the next section. 


\section{Corollaries, examples, and open problems}

To illustrate the novelty of our results, we mention the following series of corollaries and examples that are not covered by any known non-oscillation (or oscillation) criteria. At first, we provide examples to illustrate the novelty of Theorem 1.1.

Example 1 For arbitrarily given real numbers $a_{1}>0, a_{2}, b_{1}>0, b_{2}$, let us consider the equation

$$
\left(\left(a_{1}+\frac{a_{2}}{\log ^{2} t}\right)^{1-p} \Phi\left(x^{\prime}\right)\right)^{\prime}+\left(b_{1}+\sin t^{2}+\frac{b_{2}}{\log ^{2} t}\right) \frac{\Phi(x)}{t^{p}}=0,
$$

which is a special case of Eq. (1.2). The conditions in (1.3), (1.4), and (1.6) are satisfied for $R_{1}=a_{1}, S_{1}=b_{1}$, and any $\alpha \in(0,1)$, because

$$
\sup _{t \geq 1} \frac{1}{t^{\alpha}} \int_{t}^{t+t^{\alpha}}\left|\sin \tau^{2}\right| d \tau \leq 1
$$

the integral $\int^{\infty} \sin \tau^{2} d \tau$ is convergent which guarantees

$$
\sup _{t \geq \mathrm{e}} \int_{t}^{t+t^{\alpha}} \sin \tau^{2} d \tau<\infty
$$

Applying Theorems 1.1 and 4.1, we find that Eq. (5.1) is oscillatory for $a_{1}^{p-1} b_{1}>q^{-p}$ and non-oscillatory for $a_{1}^{p-1} b_{1}<q^{-p}$ and, in the limiting case $a_{1}^{p-1} b_{1}=q^{-p}$, we obtain a new result, which says that Eq. (5.1) is oscillatory for

$$
q \frac{b_{2}}{b_{1}}+p \frac{a_{2}}{a_{1}}>\frac{q^{2}}{2}, \quad \text { i.e., } \quad 2 q^{p+1} b_{2}+2 p a_{2} a_{1}^{-p}>q^{2} a_{1}^{1-p},
$$

and non-oscillatory for

$$
q \frac{b_{2}}{b_{1}}+p \frac{a_{2}}{a_{1}}<\frac{q^{2}}{2} .
$$

Indeed, Eq. (5.1) has the form of Eq. (1.2) for $r_{1}(t)=a_{1}, r_{2}(t)=a_{2}, s_{1}(t)=b_{1}+\sin t^{2}$, and $s_{2}(t)=b_{2}$, where

$$
\frac{1}{t^{\alpha}} \int_{t}^{t+t^{\alpha}} r_{2}(\tau) d \tau=a_{2}, \quad \frac{1}{t^{\alpha}} \int_{t}^{t+t^{\alpha}} s_{2}(\tau) d \tau=b_{2}
$$

for all $t \in[\mathrm{e}, \infty)$ and $\alpha \in(0,1)$.

Example 2 Let $a_{1}>0, b_{1}>0, a_{2}, a_{3}, b_{2}, b_{3}, b_{4} \in \mathbb{R}$, and $a, b, c, d \neq 0$. The half-linear equation

$$
\begin{aligned}
& \left(\left(\frac{\log ^{2} t}{a_{1} \log ^{2} t+a_{2}+a_{3} \arctan (\sin (a t))}\right)^{2}\left|x^{\prime}\right| x^{\prime}\right)^{\prime} \\
& \quad+\left(b_{1}+b_{2} \sin (b \sqrt{t})+b_{3} \sin (c t)+\frac{b_{4}+\sin (d t)}{\log ^{2} t}\right) \frac{|x| x}{t^{3}}=0
\end{aligned}
$$


takes the form of Eq. (1.2) for $p=3$ and

$$
\begin{aligned}
& r_{1}(t)=a_{1}, \quad r_{2}(t)=a_{2}+a_{3} \arctan (\sin (a t)), \\
& s_{1}(t)=b_{1}+b_{2} \sin (b \sqrt{t})+b_{3} \sin (c t), \quad s_{2}(t)=b_{4}+\sin (d t) .
\end{aligned}
$$

For any $\alpha \in(1 / 2,1)$, based on the inequalities

$$
\limsup _{t \rightarrow \infty} \frac{1}{t^{\alpha}} \int_{t}^{t+t^{\alpha}}|\sin (b \sqrt{\tau})| d \tau \leq 1
$$

and

$$
\begin{aligned}
\left|\frac{1}{t^{\alpha}} \int_{t}^{t+t^{\alpha}} \sin (b \sqrt{\tau}) d \tau\right| & \leq \frac{1}{t^{\alpha}}\left|\left[\frac{2 \sin (b \sqrt{\tau})-2 b \sqrt{\tau} \cos (b \sqrt{\tau})}{b^{2}}\right]_{t}^{t+t^{\alpha}}\right| \\
& \leq \frac{1}{b^{2} t^{\alpha}}\left(4+2 b\left(\sqrt{t+t^{\alpha}}+\sqrt{t}\right)\right)<\frac{1}{\log ^{3} t}
\end{aligned}
$$

for all large $t$, it is easy to verify conditions (1.3), (1.4), and (1.6) for large $t$. Hence, we can apply Theorem 1.1. In conclusion, Theorems 1.1 and 4.1 give the oscillation of Eq. (5.2) if $3^{3} a_{1}^{2} b_{1}>2^{3}$ and the non-oscillation if $3^{3} a_{1}^{2} b_{1}<2^{3}$. In addition, in the threshold case $3^{3} a_{1}^{2} b_{1}=2^{3}$, which is studied in this paper, we have oscillation if

$$
4 \frac{b_{4}}{b_{1}}+8 \frac{a_{2}}{a_{1}}>3
$$

and non-oscillation if the opposite sharp inequality holds.

Example 3 For arbitrary $a>1, b>1$, and $c \in \mathbb{R}$, let us consider the equations

$$
\begin{aligned}
& (p-1)\left|x^{\prime}\right|^{p-2} x^{\prime \prime}+\frac{1}{(t+1)^{p}}\left(q^{-p}+\sin _{a} t+\sin _{b} t+\frac{c}{\log ^{2} t}\right)|x|^{p-2} x=0, \\
& (p-1)\left|x^{\prime}\right|^{p-2} x^{\prime \prime}+\frac{1}{(t+1)^{p}}\left(q^{-p}+\sin _{a} t+\cos _{b} t+\frac{c}{\log ^{2} t}\right)|x|^{p-2} x=0, \\
& (p-1)\left|x^{\prime}\right|^{p-2} x^{\prime \prime}+\frac{1}{(t+1)^{p}}\left(q^{-p}+\cos _{a} t+\cos _{b} t+\frac{c}{\log ^{2} t}\right)|x|^{p-2} x=0 .
\end{aligned}
$$

Each one of these equations takes the form of Eq. (1.2). Applying Theorem 1.1, one can show that the equations are oscillatory for $2 c>q^{1-p}$ and non-oscillatory for $2 c<q^{1-p}$; this does not follow from known criteria.

Now, we focus on Theorem 4.3. In particular, Theorem 4.3 gives a new result in the linear case (for $p=2$ ) as well. Since linear equations form a very important class of equations, we mention the corresponding new corollary explicitly.

Corollary 5.1 Consider the linear equation

$$
\left(r(t) x^{\prime}(t)\right)^{\prime}+\frac{s(t)}{t^{2}} x(t)=0
$$


where $r>0, s$ are continuous functions and (4.30) is valid for some $\alpha \in[0,1)$. If there exist $R, S>0$ satisfying $4 R S \leq 1$ and a continuous function $f:[\mathrm{e}, \infty) \rightarrow \mathbb{R}$ satisfying $\lim _{t \rightarrow \infty} f(t)=0$ for which

$$
\frac{1}{t^{\alpha}} \int_{t}^{t+t^{\alpha}} \frac{1}{r(\tau)} d \tau \leq R+\frac{f(t)}{\log ^{2} t}, \quad t \in[\mathrm{e}, \infty)
$$

and

$$
\frac{1}{t^{\alpha}} \int_{t}^{t+t^{\alpha}} s(\tau) d \tau \leq S+\frac{f(t)}{\log ^{2} t}, \quad t \in[\mathrm{e}, \infty)
$$

then Eq. (5.3) is non-oscillatory.

Proof The statement of the corollary follows from Theorem 4.1 and Theorem 4.3 for $p=2$.

Further, Corollary 5.1 is not covered by known results even in the case of constant coefficients in the main term. This case is embodied into the next corollary.

\section{Corollary 5.2 Consider the linear equation}

$$
x^{\prime \prime}(t)+\frac{s(t)}{t^{2}} x(t)=0
$$

where $s$ is a continuous function and (4.30) is valid for some $\alpha \in[0,1)$. If there exists a continuous function $f:[\mathrm{e}, \infty) \rightarrow \mathbb{R}$ satisfying $\lim _{t \rightarrow \infty} f(t)=0$ such that

$$
\frac{1}{t^{\alpha}} \int_{t}^{t+t^{\alpha}} s(\tau) d \tau \leq \frac{1}{4}+\frac{f(t)}{\log ^{2} t}, \quad t \in[\mathrm{e}, \infty),
$$

then Eq. (5.4) is non-oscillatory.

Proof See Corollary 5.1 for $r \equiv 1$ and $R=1$.

To illustrate Corollary 5.2, we can consider simple types of equations whose nonoscillation does not follow from any previously known results. See the example below.

Example 4 For the function

$$
s(t):= \begin{cases}\frac{1}{4}+\frac{t-2^{n}}{n}, & t \in\left[2^{n}, 2^{n}+n\right], n \in \mathbb{N} ; \\ \frac{1}{4}+1-\frac{t-2^{n}-n}{n}, & t \in\left(2^{n}+n, 2^{n}+2 n\right], n \in \mathbb{N} ; \\ \frac{1}{4}, & t \notin\left[2^{n}, 2^{n}+2 n\right], n \in \mathbb{N},\end{cases}
$$

we consider Eq. (5.4). For any $\alpha \in(0,1)$, we have

$$
\lim _{t \rightarrow \infty} \frac{1}{t^{\alpha}} \int_{t}^{t+t^{\alpha}}|s(\tau)| d \tau=\limsup _{n \rightarrow \infty} \frac{1}{2^{\alpha n}} \int_{2^{n}}^{2^{n}+2^{\alpha n}} s(\tau) d \tau=\frac{1}{4},
$$


which gives (4.30). In addition, we obtain

$$
\frac{1}{t^{\alpha}} \int_{t}^{t+t^{\alpha}} s(\tau) d \tau \leq \frac{1}{4}+\frac{n+1}{t^{\alpha}}
$$

if $\alpha \in(0,1)$ and $t \in\left[2^{n}, 2^{n+1}\right], n \in \mathbb{N}$. Thus, if we put

$$
f(t):=\log ^{2} t \frac{1+\log _{2} t}{t^{\alpha}}, \quad t \in[\mathrm{e}, \infty)
$$

then $\lim _{t \rightarrow \infty} f(t)=0$ and (see (5.7))

$$
\frac{1}{t^{\alpha}} \int_{t}^{t+t^{\alpha}} s(\tau) d \tau \leq \frac{1}{4}+\frac{f(t)}{\log ^{2} t}, \quad t \in[\mathrm{e}, \infty)
$$

for any $\alpha \in(0,1)$. Further, we have (5.5). Altogether, Corollary 5.2 states that Eq. (5.4) is non-oscillatory for $s$ defined by (5.6). We repeat that the non-oscillation of Eq. (5.4) does not follow from any previously known results (or their combinations).

Although the aim of this paper is to prove Theorem 4.3, we obtain a more general result, which implies new criteria also for perturbed equations. Such a new corollary is as follows.

Corollary 5.3 Consider the half-linear equation

$$
\left(\left(1+\frac{r(t)}{\log ^{2} t}\right)^{1-p} \Phi\left(x^{\prime}\right)\right)^{\prime}+\left(q^{-p}+\frac{s(t)}{\log ^{2} t}\right) \frac{\Phi(x)}{t^{p}}=0
$$

where $r$, s are continuous functions and

$$
1+\frac{r(t)}{\log ^{2} t}>0, \quad t \in[\mathrm{e}, \infty)
$$

Assume that there exists $\alpha \in[0,1)$ with

$$
\limsup _{t \rightarrow \infty} \frac{1}{t^{\alpha}} \int_{t}^{t+t^{\alpha}}|r(\tau)| d \tau<\infty, \quad \limsup _{t \rightarrow \infty} \frac{1}{t^{\alpha}} \int_{t}^{t+t^{\alpha}}|s(\tau)| d \tau<\infty .
$$

(a) If

$$
\frac{1}{t^{\alpha}} \int_{t}^{t+t^{\alpha}} r(\tau) d \tau \geq R, \quad \frac{1}{t^{\alpha}} \int_{t}^{t+t^{\alpha}} s(\tau) d \tau \geq S
$$

for all large $t$ and some $R, S \in \mathbb{R}$ and

$$
q^{p+1} S+p R>\frac{q^{2}}{2}
$$

then Eq. (5.8) is oscillatory.

(b) If

$$
\frac{1}{t^{\alpha}} \int_{t}^{t+t^{\alpha}} r(\tau) d \tau \leq R, \quad \frac{1}{t^{\alpha}} \int_{t}^{t+t^{\alpha}} s(\tau) d \tau \leq S
$$


for all large $t$ and some $R, S \in \mathbb{R}$ and

$$
q^{p+1} S+p R<\frac{q^{2}}{2}
$$

then Eq. (5.8) is non-oscillatory.

Proof It suffices to use Theorem 1.1 for $R_{1}=1, S_{1}=q^{-p}, R_{2}=R, S_{2}=S, r_{1}(t)=1, s_{1}(t)=q^{-p}$, $r_{2}(t)=r(t)$, and $s_{2}(t)=s(t)$.

Corollary 5.3 is not covered by known results even for the Riemann-Weber type of (linear and half-linear) equations. Hence, we mention the next corollaries and examples.

\section{Corollary 5.4 Consider the equation}

$$
\left(\Phi\left(x^{\prime}\right)\right)^{\prime}+\left(q^{-p}+\frac{s(t)}{\log ^{2} t}\right) \frac{\Phi(x)}{t^{p}}=0,
$$

where $s$ is a continuous function such that (4.30) holds for some $\alpha \in[0,1)$.

(I) If

$$
\liminf _{t \rightarrow \infty} \frac{1}{t^{\alpha}} \int_{t}^{t+t^{\alpha}} s(\tau) d \tau>\frac{q^{1-p}}{2}
$$

then Eq. (5.9) is oscillatory.

(II) If

$$
\limsup _{t \rightarrow \infty} \frac{1}{t^{\alpha}} \int_{t}^{t+t^{\alpha}} s(\tau) d \tau<\frac{q^{1-p}}{2}
$$

then Eq. (5.9) is non-oscillatory.

Proof The corollary follows from Theorem 1.1 for coefficients $r_{1}(t)=1, r_{2}(t)=0, s_{1}(t)=$ $q^{-p}, s_{2}(t)=s(t)$.

Example 5 Let $a, b \in \mathbb{R}$ be arbitrary. Similarly as in Example 4 (see (5.6)), we define the function $s:[8, \infty) \rightarrow \mathbb{R}$ by the formula

$$
s(t):= \begin{cases}a+b \sin \frac{\pi\left(t-8^{n}\right)}{4^{n}}, & t \in\left[8^{n}, 8^{n}+4^{n}\right], n \in \mathbb{N} \\ a, & t \in\left(8^{n}+4^{n}, 8^{n+1}\right), n \in \mathbb{N} .\end{cases}
$$

Let us consider Eq. (5.9) for this function $s$ and $t \geq 8$. Clearly,

$$
\limsup _{t \rightarrow \infty} \frac{1}{t^{\alpha}} \int_{t}^{t+t^{\alpha}}|s(\tau)| d \tau \leq|a|+|b|<\infty
$$


for any $\alpha \in[0,1)$, i.e., (4.30) is fulfilled. Now, we consider $\alpha>2 / 3$. For example, we choose $\alpha=3 / 4$. Then we obtain

$$
\begin{aligned}
\liminf _{t \rightarrow \infty} \frac{1}{\sqrt[4]{t^{3}}} \int_{t}^{t+\sqrt[4]{t^{3}}} s(\tau) d \tau & \geq \lim _{n \rightarrow \infty} \frac{1}{\sqrt[4]{8^{3 n}}}\left(\int_{8^{n}}^{8^{n}+4^{n}}-|b| d \tau+\int_{8^{n}}^{8^{n}+\sqrt[4]{8^{3 n}}} a d \tau\right) \\
& =\lim _{n \rightarrow \infty} a-|b| \frac{4^{n}}{\sqrt[4]{8^{3 n}}}=\lim _{n \rightarrow \infty} a-|b|\left(\frac{4}{4 \sqrt[4]{2}}\right)^{n}=a
\end{aligned}
$$

and

$$
\begin{aligned}
\limsup _{t \rightarrow \infty} \frac{1}{\sqrt[4]{t^{3}}} \int_{t}^{t+\sqrt[4]{t^{3}}} s(\tau) d \tau & \leq \lim _{n \rightarrow \infty} \frac{1}{\sqrt[4]{8^{3 n}}}\left(\int_{8^{n}}^{8^{n}+4^{n}}|b| d \tau+\int_{8^{n}}^{8^{n}+\sqrt[4]{8^{3 n}}} a d \tau\right) \\
& =\lim _{n \rightarrow \infty} a+|b| \frac{4^{n}}{\sqrt[4]{8^{3 n}}}=a .
\end{aligned}
$$

Thus,

$$
\lim _{t \rightarrow \infty} \frac{1}{\sqrt[4]{t^{3}}} \int_{t}^{t+\sqrt[4]{t^{3}}} s(\tau) d \tau=a
$$

Applying Corollary 5.4 (see (5.10) and (5.11)) and considering (5.12), we obtain the oscillation of the considered equation for $2 a>q^{1-p}$ and its non-oscillation for $2 a<q^{1-p}$.

Corollary 5.5 Consider the linear equation

$$
x^{\prime \prime}+\left(\frac{1}{4}+\frac{s(t)}{\log ^{2} t}\right) \frac{x}{t^{2}}=0,
$$

where $s$ is a continuous function satisfying (4.30) for some $\alpha \in[0,1)$.

(a) If the inequality

$$
\frac{1}{t^{\alpha}} \int_{t}^{t+t^{\alpha}} s(\tau) d \tau>\frac{1}{4}+\varepsilon
$$

holds for all large t and some $\varepsilon>0$, then Eq. (5.13) is oscillatory.

(b) If the inequality

$$
\frac{1}{t^{\alpha}} \int_{t}^{t+t^{\alpha}} s(\tau) d \tau<\frac{1}{4}-\varepsilon
$$

holds for all large $t$ and some $\varepsilon>0$, then Eq. (5.13) is non-oscillatory.

Proof It suffices to consider Corollary 5.4 for $p=2$.

Example 6 Let $a \in \mathbb{R}$ and $\alpha \in(1 / 3,1)$ be arbitrary and let $b:[\mathrm{e}, \infty) \rightarrow \mathbb{R}$ be an arbitrary continuous function such that

$$
\limsup _{t \rightarrow \infty} \frac{1}{t^{\alpha}} \int_{t}^{t+t^{\alpha}} \sqrt[3]{\tau}|b(\tau)| d \tau<\infty .
$$


We apply Corollary 5.5 to the equation

$$
x^{\prime \prime}+\left(\frac{1}{4}+\frac{a+\arctan \left(t^{3}-2 t^{2}+1\right)+b(t) \sqrt[3]{t} \cos t+\frac{t^{5}}{t^{5}+6}}{\log (t+1) \log t}\right) \frac{x}{t^{2}}=0,
$$

i.e., for Eq. (5.13) with

$$
s(t)=\left(a+\arctan \left(t^{3}-2 t^{2}+1\right)+b(t) \sqrt[3]{t} \cos t+\frac{t^{5}}{t^{5}+6}\right) \frac{\log t}{\log (t+1)} .
$$

Since

$$
\lim _{t \rightarrow \infty} \arctan \left(t^{3}-2 t^{2}+1\right)=\frac{\pi}{2}, \quad \lim _{t \rightarrow \infty} \frac{t^{5}}{t^{5}+6}=\lim _{t \rightarrow \infty} \frac{\log t}{\log (t+1)}=1,
$$

and

$$
-\frac{2 \sqrt[3]{t+t^{\alpha}}}{t^{\alpha}} \leq \frac{1}{t^{\alpha}} \int_{t}^{t+t^{\alpha}} \sqrt[3]{\tau} \cos \tau d \tau \leq \frac{2 \sqrt[3]{t+t^{\alpha}}}{t^{\alpha}}
$$

for all large $t$, we have

$$
\lim _{t \rightarrow \infty} \frac{1}{t^{\alpha}} \int_{t}^{t+t^{\alpha}} s(\tau) d \tau=a+\frac{\pi}{2}+1
$$

To use Corollary 5.5, it remains to verify (4.30). Of course, (5.16), (5.18), and (5.19) guarantee that (4.30) is fulfilled. Considering (5.20) together with (5.14) and (5.15), Corollary 5.5 says that Eq. (5.17) is oscillatory if $4 a+2 \pi+3>0$, and non-oscillatory if $4 a+2 \pi+3<0$, i.e., if $a<-2.320796326794896619 \ldots$

In Example 6, one can see that it is possible to use the presented results asymptotically (also for other types of equations). It is formulated explicitly below in two new corollaries. Note that, for simplicity, we demonstrate such types of results only in a very special case. To prove Corollary 5.6 below, we need the following lemma.

Lemma 5.1 Let a function $F:[\mathrm{e}, \infty) \rightarrow \mathbb{R}$ be such that it can be expressed as a finite sum of continuous periodic functions. Then there exists a constant $E(F)>0$ such that the inequality

$$
\left|\frac{1}{\sqrt{t}} \int_{t}^{t+\sqrt{t}} F(\tau) d \tau-\bar{F}\right| \leq \frac{E(F)}{\sqrt{t}}
$$

holds for all $t \in[\mathrm{e}, \infty)$.

Proof See [39, Lemma 3.5].

Corollary 5.6 For an arbitrary $p>1$, consider continuous functions $f_{1}, f_{2}, f_{3}:[\mathrm{e}, \infty) \rightarrow \mathbb{R}$ such that

$$
\lim _{t \rightarrow \infty} \frac{f_{1}(t)}{\log ^{2} t}=\lim _{t \rightarrow \infty} \frac{f_{2}(t)}{\log ^{2} t}=\lim _{t \rightarrow \infty} \frac{f_{3}(t)}{t^{p}}=1
$$


and the half-linear differential equation

$$
\left(\left(1+r_{1}(t)+\frac{r_{2}(t)}{f_{1}(t)}\right)^{1-p} \Phi\left(x^{\prime}\right)\right)^{\prime}+\left(q^{-p}+s_{1}(t)+\frac{s_{2}(t)}{f_{2}(t)}\right) \frac{\Phi(x)}{f_{3}(t)}=0
$$

where $r_{1}, s_{1}$ are finite sums of continuous periodic functions, $r_{2}, s_{2}$ are continuous functions such that

$$
1+r_{1}(t)+\frac{r_{2}(t)}{f_{1}(t)}>0, \quad t \in[\mathrm{e}, \infty)
$$

the mean values $\overline{r_{1}}, \overline{s_{1}}$ are zero, and the inequalities

$$
\limsup _{t \rightarrow \infty} \frac{1}{\sqrt{t}} \int_{t}^{t+\sqrt{t}}\left|r_{2}(\tau)\right| d \tau<\infty, \quad \limsup _{t \rightarrow \infty} \frac{1}{\sqrt{t}} \int_{t}^{t+\sqrt{t}}\left|s_{2}(\tau)\right| d \tau<\infty
$$

hold. Let $R, S \in \mathbb{R}$.

(a) If

$$
\frac{1}{\sqrt{t}} \int_{t}^{t+\sqrt{t}} r_{2}(\tau) d \tau \geq R, \quad \frac{1}{\sqrt{t}} \int_{t}^{t+\sqrt{t}} s_{2}(\tau) d \tau \geq S
$$

for all large $t$ and $2 q^{p+1} S+2 p R>q^{2}$, then Eq. (5.21) is oscillatory.

(b) If

$$
\frac{1}{\sqrt{t}} \int_{t}^{t+\sqrt{t}} r_{2}(\tau) d \tau \leq R, \quad \frac{1}{\sqrt{t}} \int_{t}^{t+\sqrt{t}} s_{2}(\tau) d \tau \leq S
$$

for all large $t$ and $2 q^{p+1} S+2 p R<q^{2}$, then Eq. (5.21) is non-oscillatory.

Proof The corollary is a consequence of Theorem 1.1, where it suffices to apply Lemma 5.1 and to put $\alpha=1 / 2, R_{1}=1$, and $S_{1}=q^{-p}$.

Corollary 5.6 gives a new result for $p=2$ as well. This new result is formulated for constant coefficients in place of $r_{2}, s_{2}$.

Corollary 5.7 Let $R, S \in \mathbb{R}$ and let $f_{1}, f_{2}, f_{3}:[\mathrm{e}, \infty) \rightarrow \mathbb{R}$ be arbitrary continuous functions satisfying

$$
\lim _{t \rightarrow \infty} \frac{f_{1}(t)}{\log ^{2} t}=\lim _{t \rightarrow \infty} \frac{f_{2}(t)}{\log ^{2} t}=\lim _{t \rightarrow \infty} \frac{f_{3}(t)}{t^{2}}=1 .
$$

Consider the linear differential equation

$$
\left(\frac{f_{1}(t)}{f_{1}(t)+f_{1}(t) r(t)+R} x^{\prime}\right)^{\prime}+\left(\frac{1}{4}+s(t)+\frac{S}{f_{2}(t)}\right) \frac{x}{f_{3}(t)}=0,
$$

where $r>0$, s are finite sums of continuous periodic functions with the property that $\bar{r}=\bar{s}=0$.

(a) If $4 S+R>1$, then Eq. (5.22) is oscillatory. 
(b) If $4 S+R<1$, then Eq. (5.22) is non-oscillatory.

Proof See Corollary 5.6 for $p=2, r_{1}(t)=r(t), r_{2}(t)=R, s_{1}(t)=s(t), s_{2}(t)=S$.

Example 7 For $a, b \in \mathbb{R}$, we can illustrate Corollary 5.7, e.g., by the equations

$$
\begin{aligned}
& \left(\frac{\log ^{2} t}{a+(1+\sin t) \log ^{2} t} x^{\prime}\right)^{\prime}+\left(\frac{1}{4}\left(1+\sin t+\frac{b}{\log ^{2}(t+\sin t)}\right)\right) \frac{x}{t^{2}+2}=0, \\
& \left(\frac{\log ^{2} t}{a+\log ^{2} t} x^{\prime}\right)^{\prime}+\left(\frac{1}{4}\left(1+\cos t+\frac{b}{\log ^{2} t}\right)\right) \frac{x}{t(t+\sin t)}=0, \\
& \left(\frac{\log ^{2}(t+1)}{a+\log ^{2}(t+1)} x^{\prime}\right)^{\prime}+\left(\frac{1}{4}\left(1+\sin t \cos t+\frac{b}{\log t \log (t+\sqrt[3]{t}+1)}\right)\right) \frac{t^{2} x}{t^{4}+1}=0, \\
& \left(\left(1+\frac{a}{\log ^{2}(t+\sqrt{t} \sin t)}\right)^{-1} x^{\prime}\right)^{\prime}+\left(1+\sin t+\frac{b}{\log ^{2} t}\right) \frac{x}{4(t+1)^{2}}=0, \\
& \left(\left(1+\frac{a}{\log ^{2} t}\right)^{-1} x^{\prime}\right)^{\prime}+\left(\frac{1}{4}+\sin t+\sin (\sqrt{2} t)+\frac{b}{4 \log ^{2} t}\right) \frac{x}{t(t+1)}=0, \\
& \left(\left(1+\sin (\sqrt{2} t)+\frac{a}{\log ^{2}(t+1)}\right)^{-1} x^{\prime}\right)^{\prime}+\left(1+\sin (\sqrt{3} t)+\frac{b}{\log ^{2}(t-1)}\right) \frac{x}{4 \sqrt[4]{t^{8}+1}}=0 .
\end{aligned}
$$

One can easily verify that each one of the above equations is oscillatory for $a+b>1$ and non-oscillatory for $a+b<1$. To the best of our knowledge, the oscillatory behaviors of the above equations are not covered by any previously known criteria.

We end this paper by the following series of open problems which are connected to the research presented in this paper. Some of them are indicated in the Introduction; we describe them in detail here.

(I) Non-oscillation, principal solution, and boundary value problems. As claimed in the Introduction, non-oscillatory half-linear equations have important applications in boundary value problems on non-compact intervals. It is an open problem to study asymptotic properties of principal solutions of Euler type equations (1.2) and (4.1) under the nonoscillation condition mentioned in Theorem 4.2, (b). Along with this, it is of interest to apply these results to obtain effective criteria for the non-oscillation of principal solutions of some auxiliary half-linear equations that are used to find upper (lower) bounds for solutions of some boundary value problems on non-compact intervals by using a similar approach as in $[7,8]$.

(II) Discretization. Since the qualitative theory of difference equations follows the continuous theory frequently, the second open problem is devoted to the equations of the form

$$
\Delta\left(r_{k} \Phi\left(\Delta x_{k}\right)\right)+\frac{s_{k}}{k^{p}} \Phi\left(x_{k+1}\right)=0
$$

where $\Delta x_{k}=x_{k+1}-x_{k}$ is the forward difference of the sequence $\left\{x_{k}\right\}$. As we have already mentioned in the Introduction, some results concerning (non-perturbed) difference equations are already available (see, e.g., [36] or [34] and the references cited therein). The natural continuation is to extend known results to perturbed equations. Unfortunately, there is a problem with the method. Let us describe this problem thoroughly. 
In this paper, we started with the Riccati technique. We recall that the core of this method is the equivalence of the non-oscillation of a studied equation and the existence of a solution of an associated Riccati type differential equation (or inequality) on an interval $[T, \infty)$ (see, e.g., $[26-28,30]$ ). The standard Riccati technique and its variations (where the adapted Riccati equation (3.3) applied in this paper belongs) are used usually in the theory of non-perturbed equations. The technique itself is robust, clear, and straightforward but its "resolution" is not as precise as is needed for an analysis of the critical case and perturbed equations.

Hence, we combine the adapted Riccati equation with the Prüfer technique and involve the averaging method. Then we use the equivalence of the non-oscillation of the given equation and the boundedness of the Prüfer angle (for variations of such approach, see, e.g., $[17,33,39,45])$.

Getting back to difference equations, the first approach (the Riccati technique) is available and frequently applied. The second method which involves the Prüfer transformation is available only in a "weak" version, which is a consequence of problems with the chain rule in the difference calculus (see [4]). Therefore, the acquisition of results concerning the conditional oscillation for difference equations with perturbations (or solve the critical case) remains a challenging open problem.

(III) Time scales. The third direction is towards dynamic equations on time scales, i.e., to equations of the form

$$
\left(r(t) \Phi\left(x^{\Delta}\right)\right)^{\Delta}+\frac{s(t)}{t^{p}} \Phi\left(x^{\sigma}\right)=0,
$$

where $x^{\Delta}$ and $x^{\sigma}$ denote the delta derivative and the forward jump operator applied to the function $x$, respectively. For more details, we refer, e.g., to [5]. Roughly speaking, the time scale calculus is a unification of the continuous and discrete calculus but a time scale itself is an arbitrary non-empty closed subset of real numbers. It means that much more cases are covered than only $\mathbb{R}$ and $\mathbb{Z}$. It has lead to the importance of dynamic equations in natural sciences, economy, and informatics and also to high interest of researchers. As well as in the discrete case (which is a special case of time scales), some results are already known (see, e.g., [38] with references therein). Of course, the problem with the fully functional Prüfer transformation is present as well. Therefore, the description from point (II) is valid also for dynamic equations on time scales. Moreover (see [50]), we note that the critical oscillation constant may be dependent on the graininess (a function that measures the distance between two consecutive points of the given time scale; it is identically zero for $\mathbb{R}$ and one for $\mathbb{Z}$ ).

(IV) Modified equations. Since conditionally oscillatory equations are very useful, a natural direction of research is to find another types of equations that are conditionally oscillatory. As we have already mentioned in the Introduction, such equations are ideal for testing oscillatory properties of other equations via many comparison theorems. However, the form of the coefficients of known conditionally oscillatory equations may not be suitable for testing of certain equations. More precisely, let us consider Eq. (2.4), where the presence of $t^{-p}$ in the potential term may be an obstacle. It is proved in [14] that the equation

$$
\left(t^{\beta-1} r(t) \Phi\left(x^{\prime}\right)\right)^{\prime}+\frac{s(t)}{t^{p-\beta+1}} \Phi(x)=0, \quad \beta \neq p
$$


is conditionally oscillatory. The idea is to find other forms of differential equations and their perturbations that preserve the behavior of conditional oscillation. In this direction, we recall Theorem 2.3 .

We remark that Eq. (5.23) with $\beta=p$ is not conditionally oscillatory, which can be simply verified on the case of constant coefficients $r$, s. In this case (see [35]), the form of the corresponding conditionally oscillatory equation is

$$
\left(t^{p-1} r(t) \Phi\left(x^{\prime}\right)\right)^{\prime}+\frac{s(t)}{t \log ^{p} t} \Phi(x)=0
$$

and the conditionally oscillatory perturbed equation is

$$
\left(t^{p-1}\left(r_{1}(t)+\frac{r_{2}(t)}{\log ^{2} t}\right)^{1-p} \Phi\left(x^{\prime}\right)\right)^{\prime}+\frac{1}{t \log ^{p} t}\left(s_{1}(t)+\frac{s_{2}(t)}{\log ^{2} t}\right) \Phi(x)=0 .
$$

Continuation in this research direction has a potential to enlarge the set of conditionally oscillatory equations.

(V) Non-linear equations. Besides the applications within linear and half-linear equations (and "real world" applications in natural sciences or economics), half-linear equations can be considered a starting point to research in the field of non-linear equations. A natural way involves equations with the combination of two $p$-Laplacians such as

$$
\left(r(t) \Phi_{a}\left(x^{\Delta}\right)\right)^{\Delta}+s(t) \Phi_{b}\left(x^{\sigma}\right)=0
$$

where $\Phi_{a}$ and $\Phi_{b}$ stand for $p$-Laplacian with $p=a$ and $p=b$, respectively. Then one can proceed to equations of the form

$$
\left(r(t) \Phi\left(x^{\Delta}\right)\right)^{\Delta}+s(t) g\left(x^{\sigma}\right)=0,
$$

where $g$ satisfies the sign condition $x g(x)>0$ whenever $x \neq 0$. See, e.g., [55-57, 59].

(VI) Partial differential equations. Since half-linear equations are in fact PDE's with one dimensional $p$-Laplacian, another natural way of research is towards elliptic partial differential equations in the form

$$
\operatorname{div}\left(A(x)\|\nabla u\|^{p-2} \nabla u\right)+C(x) \Phi(u)=0
$$

where $A$ is an elliptic $n \times n$ matrix function with differentiable components, $C$ is a Hölder continuous function, and $x \in \mathbb{R}^{n}$. See, e.g., $[15,18,49]$ or [28], where an example of a result for Eq. (5.24) is presented using a theorem about conditionally oscillatory half-linear equations.

(VII) General coefficients. Whenever a result containing some restrictions on coefficients is proved, the main question is whether the limitations on coefficients can be removed or weakened at least. We conclude that the results of this paper cannot be significantly extended in this direction. We can illustrate this on Eq. (4.29), which is proved to be nonoscillatory in the critical case (see Theorem 4.3). If we allow functions $r, s$ to be almost periodic, we can construct two sets of these functions, say $r_{1}, s_{1}$ and $r_{2}, s_{2}$, such that one of the obtained equations (e.g., with $r_{1}, s_{1}$ ) is oscillatory and the second one (with $r_{2}, s_{2}$ ) 
is non-oscillatory in the critical case. Constructions that can be used for obtaining these coefficients are described in [60] (and used, e.g., in [29, 31] in the discrete case).

\section{Acknowledgements \\ Not applicable.}

\section{Funding}

The research was supported by Grant GA17-03224S of the Czech Science Foundation. The third author was supported by the Gruppo Nazionale per I'Analisi Matematica, la Probabilità e le loro Applicazioni (GNAMPA) of the Italian Istituto Nazionale di Alta Matematica (INdAM).

\section{Availability of data and materials}

Data sharing not applicable to this article as no datasets were generated or analyzed during the current study.

\section{Competing interests}

The authors declare that they have no competing interests.

\section{Authors' contributions}

All authors contributed equally to the writing of this paper. The authors read and approved the final manuscript.

\section{Author details}

${ }^{1}$ Department of Mathematics and Statistics, Faculty of Science, Masaryk University, Brno, Czech Republic. ${ }^{2}$ Department of Mathematics and Computer Science "Ulisse Dini", University of Florence, Florence, Italy.

\section{Publisher's Note}

Springer Nature remains neutral with regard to jurisdictional claims in published maps and institutional affiliations.

Received: 15 February 2019 Accepted: 20 June 2019 Published online: 05 July 2019

\section{References}

1. Agarwal, R.P., Grace, S.R., O'Regan, D.: Oscillation Theory for Second Order Linear, Half-Linear, Superlinear and Sublinear Dynamic Equations. Kluwer Academic, Dordrecht (2002)

2. Bihari, I.: An oscillation theorem concerning the half-linear differential equation of second order. Magy. Tud. Akad. Mat. Kut. Intéz. Közl. 8, 275-280 (1964)

3. Bognár, G., Došlý, O.: Half-linear eigenvalue problem: limit behavior of the first eigenvalue for shrinking interval. Bound. Value Probl. 2013, 221 (2013)

4. Bohner, M., Došlý, O.: The discrete Prüfer transformation. Proc. Am. Math. Soc. 129(9), 2715-2726 (2001)

5. Bohner, M., Peterson, A.: Dynamic Equations on Time Scales: An Introduction with Applications. Birkhäuser, Boston (2001)

6. Došlá, Z., Marini, M.: Positive decaying solutions for differential equations with phi-Laplacian. Bound. Value Probl. 2015, 95 (2015)

7. Došlá, Z., Marini, M., Matucci, S.: A Dirichlet problem on the half-line for nonlinear equations with indefinite weight. Ann. Mat. Pura Appl. 196(1), 51-64 (2017)

8. Došlá, Z., Marini, M., Matucci, S.: Global Kneser solutions to nonlinear equations with indefinite weight. Discrete Contin. Dyn. Syst., Ser. B 23(8), 3297-3308 (2018)

9. Došlá, Z., Matucci, S.: Ground state solutions to nonlinear equations with p-Laplacian. Nonlinear Anal. 184, 1-16 (2019)

10. Došlý, O.: Half-linear Euler differential equation and its perturbations. Electron. J. Qual. Theory Differ. Equ. 2016, 10 (2016) Proc. 10'th Coll. Qual. Theory Diff. Equ.

11. Došlý, O., Fišnarová, S.: Linearized Riccati technique and (non-)oscillation criteria for half-linear difference equations. Adv. Differ. Equ. 2008, Article ID 438130, 1-18 (2008)

12. Došlý, O., Funková, H.: Euler type half-linear differential equation with periodic coefficients. Abstr. Appl. Anal. 2013, Article ID 714263 (2013)

13. Došlý, O., Hasil, P.: Critical oscillation constant for half-linear differential equations with periodic coefficients. Ann. Mat Pura Appl. 190(3), 395-408 (2011)

14. Došlý, O., Jaroš, J., Veselý, M.: Generalized Prüfer angle and oscillation of half-linear differential equations. Appl. Math Lett. 64(2), 34-41 (2017)

15. Došlý, O., Mařík, R.: Nonexistence of positive solutions of PDE's with p-Laplacian. Acta Math. Hung. 90(1-2), 89-107 (2001)

16. Došlý, O., Řehák, P.: Half-Linear Differential Equations. Elsevier, Amsterdam (2005)

17. Došlý, O., Veselý, M.: Oscillation and non-oscillation of Euler type half-linear differential equations. J. Math. Anal. Appl. 429(1), 602-621 (2015)

18. Došlý, O., Yamaoka, N.: Oscillation constants for second-order ordinary differential equations related to elliptic equations with $p$-Laplacian. Nonlinear Anal. 113, 115-136 (2015)

19. Drábek, P.: Fredholm alternative for the $p$-Laplacian: yes or no? In: Function Spaces, Differential Operators and Nonlinear Analysis, Pudasjärvi, 1999, pp. 57-64. Czech. Acad. Sci., Prague (2000)

20. Elbert, Á:: The Wronskian and the half-linear differential equations. Studia Sci. Math. Hung. 15(1-3), 101-105 (1980)

21. Elbert, Á.: Oscillation and nonoscillation theorems for some nonlinear ordinary differential equations. In: Ordinary and Partial Differential Equations, Dundee, 1982. Lecture Notes in Math., vol. 964, pp. 187-212. Springer, Berlin (1982) 
22. Elbert, Á.: Asymptotic behaviour of autonomous half-linear differential systems on the plane. Studia Sci. Math. Hung. 19(2-4), 447-464 (1984)

23. Elbert, Á., Schneider, A.: Perturbations of half-linear Euler differential equation. Results Math. 37(1-2), 56-83 (2000)

24. Fišnarová, S., Mařík, R.: Oscillation criteria for neutral second-order half-linear differential equations with applications to Euler type equations. Bound. Value Probl. 2014, 83, 1-14 (2014)

25. Gesztesy, F., Ünal, M.: Perturbative oscillation criteria and Hardy-type inequalities. Math. Nachr. 189, $121-144$ (1998)

26. Hasil, P.: Conditional oscillation of half-linear differential equations with periodic coefficients. Arch. Math. 44(2), 119-131 (2008)

27. Hasil, P., Juránek, J., Veselý, M.: Adapted Riccati technique and non-oscillation of linear and half-linear equations. Appl. Math. Lett. 82, 98-105 (2018)

28. Hasil, P., Mařík, R., Veselý, M.: Conditional oscillation of half-linear differential equations with coefficients having mean values. Abstr. Appl. Anal. 2014, Article ID 258159 (2014)

29. Hasil, P., Veselý, M.: Almost periodic transformable difference systems. Appl. Math. Comput. 218(9), 5562-5579 (2012)

30. Hasil, P., Veselý, M.: Oscillation of half-linear differential equations with asymptotically almost periodic coefficients. Adv. Differ. Equ. 2013, 122, 1-15 (2013)

31. Hasil, P., Veselý, M.: Limit periodic homogeneous linear difference systems. Appl. Math. Comput. 265, 958-972 (2015)

32. Hasil, P., Veselý, M.: Non-oscillation of half-linear differential equations with periodic coefficients. Electron. J. Qual. Theory Differ. Equ. 2015, 1, 1-21 (2015)

33. Hasil, P., Veselý, M.: Non-oscillation of perturbed half-linear differential equations with sums of periodic coefficients. Adv. Differ. Equ. 2015, 190, 1-17 (2015)

34. Hasil, P., Veselý, M.: Oscillation constants for half-linear difference equations with coefficients having mean values. Adv. Differ. Equ. 2015, 210, 1-18 (2015)

35. Hasil, P., Veselý, M.: Oscillation and non-oscillation criterion for Riemann-Weber type half-linear differential equations Electron. J. Qual. Theory Differ. Equ. 2016, 59, 1-22 (2016)

36. Hasil, P., Veselý, M.: Oscillation and non-oscillation criteria for linear and half-linear difference equations. J. Math. Anal. Appl. 452(1), 401-428 (2017)

37. Hasil, P., Veselý, M.: Oscillation and non-oscillation results for solutions of perturbed half-linear equations. Math. Methods Appl. Sci. 41(9), 3246-3269 (2018)

38. Hasil, P., Veselý, M.: Oscillatory and non-oscillatory solutions of dynamic equations with bounded coefficients. Electron. J. Differ. Equ. 2018, 24, 1-22 (2018)

39. Hasil, P., Veselý, M.: Prüfer angle and non-oscillation of linear equations with quasiperiodic data. Monatshefte Math. 189(1), 101-124 (2019)

40. Hasil, P., Vítovec, J.: Conditional oscillation of half-linear Euler-type dynamic equations on time scales. Electron. J. Qual. Theory Differ. Equ. 2015, 6, 1-24 (2015)

41. Hongyo, A., Yamaoka, N.: General solutions for second-order linear difference equations of Euler type. Opusc. Math. 37(3), 389-402 (2017)

42. Jaroš, J., Veselý, M.: Conditional oscillation of Euler type half-linear differential equations with unbounded coefficients. Studia Sci. Math. Hung. 53(1), 22-41 (2016)

43. Kneser, A.: Untersuchungen über die reellen Nullstellen der Integrale linearer Differentialgleichungen. Math. Ann. 42(3), 409-435 (1893)

44. Krüger, H.: On perturbations of quasiperiodic Schrödinger operators. J. Differ. Equ. 249(6), 1305-1321 (2010)

45. Krüger, H., Teschl, G.: Effective Prüfer angles and relative oscillation criteria. J. Differ. Equ. 245(12), 3823-3848 (2008)

46. Krüger, H., Teschl, G.: Relative oscillation theory for Sturm-Liouville operators extended. J. Funct. Anal. 254(6), $1702-1720(2008)$

47. Krüger, H., Teschl, G.: Relative oscillation theory, weighted zeros of the Wronskian, and the spectral shift function. Commun. Math. Phys. 287(2), 613-640 (2009)

48. Li, T., Baculíková, B., Džurina, J.: Oscillatory behavior of second-order nonlinear neutral differential equations with distributed deviating arguments. Bound. Value Probl. 2014, 68, 1-15 (2014)

49. Mařík, R.: Ordinary differential equations in the oscillation theory of partial half-linear differential equation. J. Math. Anal. Appl. 338(1), 194-208 (2008)

50. Řehák, P.: A critical oscillation constant as a variable of time scales for half-linear dynamic equations. Math. Slovaca 60(2), 237-256 (2010)

51. Řehák, P., Yamaoka, N.: Oscillation constants for second-order nonlinear dynamic equations of Euler type on time scales. J. Differ. Equ. Appl. 23(11), 1884-1900 (2017)

52. Schmidt, K.M.: Oscillation of perturbed Hill equation and lower spectrum of radially periodic Schrödinger operators in the plane. Proc. Am. Math. Soc. 127, 2367-2374 (1999)

53. Schmidt, K.M.: Critical coupling constant and eigenvalue asymptotics of perturbed periodic Sturm-Liouville operators. Commun. Math. Phys. 211, 465-485 (2000)

54. Sugie, J.: Nonoscillation criteria for second-order nonlinear differential equations with decaying coefficients. Math. Nachr. 281(11), 1624-1637 (2008)

55. Sugie, J., Hara, T.: Nonlinear oscillations of second order differential equations of Euler type. Proc. Am. Math. Soc. 124(10), 3173-3181 (1996)

56. Sugie, J., Kita, K.: Oscillation criteria for second order nonlinear differential equations of Euler type. J. Math. Anal. Appl. 253(2), 414-439 (2001)

57. Sugie, J., Matsumura, K.: A nonoscillation theorem for half-linear differential equations with periodic coefficients. Appl. Math. Comput. 199(2), 447-455 (2008)

58. Sugie, J., Onitsuka, M.: A non-oscillation theorem for nonlinear differential equations with p-Laplacian. Proc. R. Soc. Edinb. A 136(3), 633-647 (2006)

59. Sugie, J., Yamaoka, N.: Comparison theorems for oscillation of second-order half-linear differential equations. Acta Math. Hung. 111(1-2), 165-179 (2006)

60. Veselý, M.: Construction of almost periodic functions with given properties. Electron. J. Differ. Equ. 2011, 29 (2011)

61. Veselý, M., Hasil, P.: Conditional oscillation of Riemann-Weber half-linear differential equations with asymptotically almost periodic coefficients. Studia Sci. Math. Hung. 51(3), 303-321 (2014) 
62. Vítovec, J.: Critical oscillation constant for Euler-type dynamic equations on time scales. Appl. Math. Comput. 243, 838-848 (2014)

63. Yamaoka, N.: Oscillation criteria for second-order nonlinear difference equations of Euler type. Adv. Differ. Equ. 2012, 218, 1-14 (2012)

Submit your manuscript to a SpringerOpen ${ }^{\mathcal{O}}$ journal and benefit from:

- Convenient online submission

$\checkmark$ Rigorous peer review

- Open access: articles freely available online

- High visibility within the field

- Retaining the copyright to your article

Submit your next manuscript at $\gg$ springeropen.com 